\title{
Inflation in AdS/CFT
}

\author{
Ben Freivogel $^{a, b}$, Veronika E. Hubeny $^{b, c}$, Alexander Maloney ${ }^{a, d}$, \\ Rob Myers ${ }^{e, f}$, Mukund Rangamani ${ }^{b, c}$, and Stephen Shenker ${ }^{a}$ \\ a Department of Physics, Stanford University, Stanford, CA 94305, USA \\ ${ }^{b}$ Department of Physics 83 Theoretical Physics Group, LBNL, Berkeley, CA 94720, USA \\ ${ }^{c}$ Department of Mathematical Sciences, University of Durham, Durham DH1 3LE, UK \\ $d$ Stanford Linear Accelerator Center, Menlo Park, CA 94025, USA \\ e Perimeter Institute for Theoretical Physics, Waterloo, Ontario, N2L 2Y5, Canada \\ ${ }^{f}$ Department of Physics, University of Waterloo, Waterloo, Ontario, N2L 3G1, Canada
}

\begin{abstract}
We study the realization of inflation within the AdS/CFT correspondence. We assume the existence of a string landscape containing at least one stable AdS vacuum and a (nearby) metastable de Sitter state. Standard arguments imply that the bulk physics in the vicinity of the AdS minimum is described by a boundary CFT. We argue that large enough bubbles of the dS phase, including those able to inflate, are described by mixed states in the CFT. Inflating degrees of freedom are traced over and do not appear explicitly in the boundary description. They nevertheless leave a distinct imprint on the mixed state. Analytic continuation allows us, in principle, to recover a large amount of nonperturbatively defined information about the inflating regime. Our work also shows that no scattering process can create an inflating region, even by quantum tunneling, since a pure state can never evolve into a mixed state under unitary evolution.
\end{abstract}

Submitted to Journal of High Energy Physics

October 2005

\footnotetext{
freivogel@berkeley.edu, veronika.hubeny@durham.ac.uk, maloney@slac.stanford.edu, rmyers@perimeterinstitute.ca, mukund.rangamani@durham.ac.uk, sshenker@stanford.edu

SU-ITP-05-27 UCB-PTH-05/30 LBNL-58913 DCPT-05/45 SLAC-PUB-11505
}

Work supported in part by Department of Energy contract DE-AC02-76SF00515 


\section{Contents}

1. Introduction . . . . . . . . . . . . . . . . . . . . . . . . . . . . . . . . . 1

2. Inflation in asymptotically AdS spacetimes . . . . . . . . . . . . . . . . . . . . 3

2.1. Thin domain wall constructions . . . . . . . . . . . . . . . . . . . . . . . 5

2.2. Beyond the thin wall approximation . . . . . . . . . . . . . . . . . . . . . 13

2.3. A special parameter domain . . . . . . . . . . . . . . . . . . . . . . . . . 14

3. Properties of the boundary CFT . . . . . . . . . . . . . . . . . . . . . 15

3.1. The entropy puzzle . . . . . . . . . . . . . . . . . . . . . . . . . . . . . 16

3.2. Mixed states in asymptotically Schwarzschild-AdS geometries . . . . . . . . . . 18

3.3. Conditions for the appearance of mixed states . . . . . . . . . . . . . . . . . . 21

4. Probes of inflation in AdS/CFT . . . . . . . . . . . . . . . . . . . . . . . . . 25

4.1. Geodesics probes of domain wall spacetimes . . . . . . . . . . . . . . . . . . 26

4.2. From AdS/CFT to dS/CFT and beyond . . . . . . . . . . . . . . . . . . . 29

5. Can inflation begin by tunneling? . . . . . . . . . . . . . . . . . . . . . . . . 31

6. Discussion . . . . . . . . . . . . . . . . . . . . . . . . . . . . . . . . . . 32

Appendix A. Details of the thin wall geometries . . . . . . . . . . . . . . . . . . . 34

A.1. Effective potential and extrinsic curvatures . . . . . . . . . . . . . . . . . . 34

A.2. Thin wall trajectories . . . . . . . . . . . . . . . . . . . . . . . . . . . 35

Appendix B. False vacuum bubbles in scalar-gravity systems . . . . . . . . . . . . . 39

Appendix C. Construction allowing de Sitter $\mathcal{I}$ and $r_{d}<r_{+}$. . . . . . . . . . . . . . . . . 43

Appendix D. A pure state description of spacetimes with causally disconnected regions? . 45

Appendix E. Analyticity in Coleman-de Luccia spacetimes . . . . . . . . . . . . . . . 48

\section{Introduction}

Our current understanding of the cosmological evolution of the universe relies on the existence of an early period of inflation 1 . Recent data suggest that the universe is now undergoing another period of inflation. It is of central importance to understand this remarkable phenomenon as deeply as possible.

String theory, which currently is the only viable candidate for a theory of quantum gravity, has had partial success in describing inflationary physics. In recent years there has been dramatic progress in constructing string vacua with stabilized moduli and positive and negative cosmological constants [4, 5, 6, [, 8, 8, 9, 10, 11]. These lead to de Sitter and Antide Sitter (AdS) cosmologies, respectively. There are an enormous number of such vacua, populating what is now called the "string landscape" [12]. A small piece of the landscape, containing an AdS and a neighboring de Sitter vacuum, is sketched in Fig. 1. The richness of the landscape allows us to view the parameters of such a potential as essentially free parameters.

1 For reviews see [1, 2, 3, 3. 


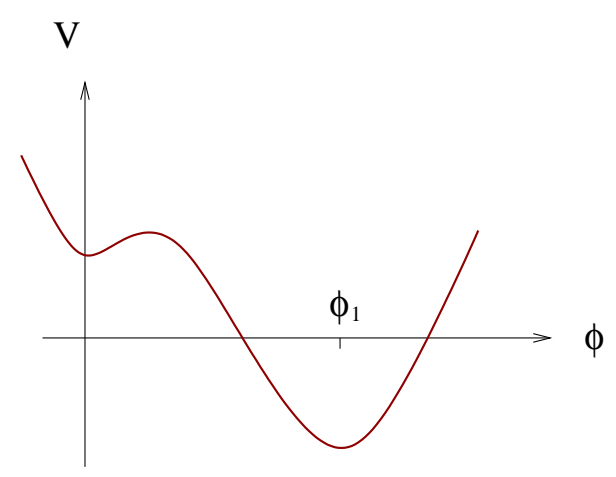

Fig. 1: A typical scalar potential appearing in string theory, with de Sitter and Anti-de Sitter minima.

These constructions do not answer many of the deep questions raised by inflation: How can inflation begin? What measure should be used on the multiverse of eternal inflation? What is the holographic description of inflation? More generally, what degrees of freedom are appropriate for a complete description of quantum gravity in this domain? A substantial amount of work has been done on these topics [13, 14, 15, 16, 17, 18, 19]. We will not be able to answer these questions in this paper, but we will try to make some progress by embedding inflation in our best understood and most powerful framework for understanding quantum gravity, the AdS/CFT correspondence 2 [21,22, 23, 24].

Consider a stable supersymmetric AdS ground state, say the one indicated in Fig. 1. The bulk correlators taken to the AdS boundary define a conformal field theory (CFT), which encodes the bulk dynamics precisely. Given that small fluctuations around the AdS minimum are captured by the CFT, it seems plausible that classical configurations corresponding to the excursions to the neighboring de Sitter minimum should be encoded in the CFT somehow. For instance, correlators of the scalar field $\phi$ describing the horizontal axis of Fig. 1 should enable one to reconstruct the effective potential3. If we can construct a region of space where $\phi$ is displaced from the AdS minimum to the dS minimum 1 , the behavior of such a bubble of false vacuum should contain some inflationary physics.

The fate of such bubbles has been investigated extensively while exploring the possibility of "creating a universe in a laboratory" 25,26,27]. We will discuss these results in more detail later, but for now we will just summarize the main points. Observed from the boundary of AdS (where the dual CFT is located), all such bubbles collapse into a black

2 The first work on this connection is [20].

3 Of course, a CFT that captures aspects of the landscape as a whole must be a complicated object indeed.

4 The finite energy excitations described by the AdS/CFT correspondence require that $\phi$ approach the AdS minimum at the AdS boundary. 
hole. If the bubble was large enough initially, an inflating region forms, but it is behind the black hole horizon. At first glance this seems discouraging. The standard AdS/CFT observables are only sensitive to physics outside the horizon. But in recent years tools have been developed, based largely on analyticity, to examine physics behind the horizon in AdS/CFT [28,29,30,31. We will discuss here how these tools enable us to observe the inflating region.

A basic obstruction to making a universe in a laboratory classically was noted by the authors of [26]. They argued using singularity theorems in general relativity that an inflating region must classically always begin in a singularity. But AdS/CFT quite comfortably describes geometries with both future and past singularities, like the eternal Schwarzschild-AdS black hole [32,33]. So the observations of [26] should not prevent us from studying inflation in AdS/CFT.

A more general worry about representing inflation in AdS/CFT is that the boundary CFT must encode a very large number of degrees of freedom describing the inflating region. Specifically, the authors of [34, 35] have pointed out that in certain situations the dS entropy of the inflating region is larger than the entropy of the Schwarzschild-AdS black hole. Notions of black hole complementarity and holography suggest that this would be hard to accommodate.

In our picture this puzzle is resolved in a simple way. We present arguments that the geometries created by large bubbles of false vacuum must be represented as mixed states in the boundary CFT. The large number of degrees of freedom in the region behind the horizon are entangled with the degrees of freedom outside the horizon, as in the HartleHawking state representation of the eternal Schwarzschild-AdS black holeb [36,32, 33]. The degrees of freedom behind the horizon are not explicitly represented; they are traced over. They can be weakly entangled, though, so that even tracing over a large number of them can yield a density matrix with entropy compatible with the black hole entropy.

Even though the inflating degrees of freedom are traced over, and hence not explicitly present, a large amount of information about them can be extracted, again by using analyticity. As an example, in the eternal Schwarzschild-AdS black hole, boundary operators on the right hand boundary can be moved to the left hand boundary by continuing in complex time. In our situation, boundary operators on the right hand AdS boundary can be moved by a suitable continuation in complex time to the de Sitter boundary at future (or past) infinity. The resulting correlators living on the boundary of de Sitter have the form that one would expect from the dS/CFT correspondence [19]. This conclusion holds classically. Quantum mechanically the de Sitter regions decay and the behavior is

5 As we review later, the eternal Schwarzschild-AdS black hole is described as a pure entangled state in the Hilbert space of two copies of the boundary CFT, each living on a separate AdS boundary. Tracing over one of these Hilbert spaces leads to a thermal density matrix in the other. 
richer, and more mysterious. These analytically continued correlators give, in principle, a non-perturbative description of this physics.

The paper is organized as follows. In Section 2 we review the construction of false vacuum bubble spacetimes in the thin wall approximation and discuss various aspects of these geometries. In Section 3 we describe the realization of these spacetimes in AdS/CFT. We consider the entropy puzzle and resolve it by presenting arguments showing that the CFT describing inflation must be in a mixed state. We also discuss the general question of which geometries are represented by mixed states. Section 4 deals with the signatures of inflating bubbles in the CFT correlation functions. We demonstrate that geodesic probes can sample the inflationary universe behind the horizon and describe the signatures that can be gleaned from this analysis. We discuss analytic continuation from the AdS to the de Sitter boundary, which in principle gives a non-perturbative description of the far future of inflation. In Section 5 we revisit the idea of "creating" a universe in the laboratory from our perspective. We argue that because the CFT dual of an inflating region is a mixed state it cannot be produced in any scattering process, including quantum tunnelling, which is described by pure state evolution. This agrees with some previous work [37,38. We end with a discussion in Section 6. Some calculations are collected in appendices.

\section{Inflation in asymptotically AdS spacetimes}

Recently, the landscape of string theory compactifications has been shown to include both Anti-de Sitter and de Sitter minima [4, $5,6,6,7,8,9,10,11]$. The theory includes domain walls interpolating between these states, so one might expect that there are asymptotically AdS spacetimes containing an inflating de Sitter region. For many classes of compactifications, the low energy theory is effectively described by gravity coupled to a scalar field in a potential, as in Fig. 1. This effective potential contains both positive and negative energy minima, with a domain wall given by field configurations interpolating between two

vacua. Hence, by choosing carefully the initial profile of the scalar field and solving the equations of motion, one can obtain asymptotically AdS spaces with inflating regions in the low energy effective gravitational theory.

Although this effective model is much simpler than the full string theory, it is nevertheless quite complicated: even solving for the exact spacetime metric requires messy numerical computations. So for much of this section we will work in the thin wall approximation, where we can write down the metric exactly. In this approximation we simply match two pieces of known spacetimes together across an infinitesimally thin 'domain wall', which obeys the appropriate junction conditions [39]. The simplest requirement for this approximation to be valid is that the width of the domain wall be less than the curvature length scales in the geometry. This is easy to arrange. More refined requirements will 
be discussed below. For spherically symmetric spacetimes joined across a spherical shell, the full configuration is likewise spherically symmetric, and may be viewed as a bubble of one spacetime inside the other spacetime. Although the metric is continuous across the bubble wall, the extrinsic curvature is discontinuous because the shell carries some energy. Einstein's equations then reduce to an effective equation of motion for this shell. We merely have to solve this equation to determine the shell's trajectory, and patch together the spacetimes across the shell.

Spherical symmetry enables us to draw two-dimensional Penrose diagrams which encode the full causal structure of the entire spacetime. In addition, knowing the metric exactly will allow us to study the behavior of the geodesics, Green's functions, etc., in these spacetimes, which will ultimately be of use in extracting information about this spacetime from its holographic dual.

We will first consider thin domain wall constructions, before broadening our discussion to include the more realistic (scalar field) set-up towards the end. We begin by discussing what types of geometries are possible, specializing mainly to a bubble of de Sitter inside Schwarzschild-AdS. After explaining the construction and categorizing the various possible cases, we focus on time-symmetric situations. As we will see, having a piece of de Sitter infinity (denoted $\mathcal{I}$ ) guarantees the existence of a de Sitter horizon; time symmetry then guarantees that its area is necessarily greater than that of the black hole horizon. As will be discussed in Section 3, this would seem to lead to an entropy paradox. There are also time asymmetric solutions with de Sitter $\mathcal{I}$ - in this case the de Sitter horizon may be larger or smaller than the black hole horizon.

We will then illustrate explicitly that one can achieve essentially the same desirable ingredients (namely de Sitter $\mathcal{I}$ hidden behind a horizon) for a scalar field in a suitably chosen potential6. The latter is chosen by hand, but motivated by the string landscape; we discuss what are reasonable landscape parameters to expect.

\subsection{Thin domain wall constructions}

We start by reviewing the procedure of patching together geometries across a thin junction in general relativity [39]. This will allow us to construct classical solutions with both AdS and de Sitter regions (including $\mathcal{I}$ ). For simplicity, we consider only spherically symmetric geometries in four dimensions - more general solutions are considered in Appendix A.

We have a spherical shell, inside of which the metric is

$$
d s_{i}^{2}=-f_{i}(r) d t_{i}^{2}+\frac{d r^{2}}{f_{i}(r)}+r^{2} d \Omega^{2},
$$

6 However, as we will see, the nature of some parts of singularities and boundaries may be altered by instabilities. 
and outside of which the metric is

$$
d s_{o}^{2}=-f_{o}(r) d t_{o}^{2}+\frac{d r^{2}}{f_{o}(r)}+r^{2} d \Omega^{2}
$$

Note that having written the metrics in a static, spherically symmetric form, we must allow for the 'time' coordinates $t_{\alpha}(\alpha=i, o)$ to be different in each region, since this coordinate need not match across the shell. On the other hand, $r$ is a physically meaningful coordinate - it measures the proper size of the spheres of a spherically symmetric spacetime - and therefore has to vary continuously across the shell. Hence we can use the same coordinate $r$ both inside and outside the shell.

The inside and outside geometries are patched together along a domain wall, with world-volume metric

$$
d s_{\text {bubble }}^{2}=-d \tau^{2}+R(\tau)^{2} d \Omega^{2} .
$$

Here $R(\tau)$ denotes the proper size of the shell as a function of its proper time $\tau$; in each part of the spacetime its trajectory is given by $r=R(\tau)$. In the thin wall approximation we take the domain wall stress tensor to be delta function localized on the wall surface. The equation of motion of the shell, which determines $R(\tau)$, then follows from two matching conditions (for a review, see e.g. [25]). First, the metric must be continuous across the domain wall. Second, the jump in extrinsic curvature across the wall is related to the stress tensor of the bubble. This implies that

$$
\sqrt{\dot{R}^{2}+f_{i}(R)}-\sqrt{\dot{R}^{2}+f_{o}(R)}=\kappa R
$$

(with the sign of the radical determined by the extrinsic curvature - see below), where $\dot{R} \equiv \frac{d R}{d \tau}$. The parameter $\kappa=4 \pi G_{N} \sigma$ is related to the domain wall tension $\sigma$. By squaring (2.4) twice, we obtain the radial equation of motion of the shell,

$$
\dot{R}^{2}+V_{\text {eff }}(R)=0
$$

with the effective potential

$$
V_{\mathrm{eff}}(r)=f_{o}(r)-\frac{\left(f_{i}(r)-f_{o}(r)-\kappa^{2} r^{2}\right)^{2}}{4 \kappa^{2} r^{2}} .
$$

Equation (2.5) describes the one-dimensional motion of a point particle of zero energy in an effective potential (2.6). Many properties of the geometry can be read off directly from the form of $V_{\text {eff }}(r)$. For example, if $V_{\text {eff }}(r) \rightarrow+\infty$ (or $V_{\text {eff }}(r) \rightarrow C>0$ ) as $r \rightarrow \infty$, the shell cannot reach the boundary. 
We should note that the equation of motion (2.5) actually does not completely determine spacetime when, as in Schwarzschild-AdS or de Sitter, $r$ is not a global coordinated. This is because the effective potential (2.6) was obtained by squaring the equation for junction conditions twice, so we have lost some sign information. In particular, 2.5 does not distinguish between different points with the same value of $r$. To fix this, we must take into account the extrinsic curvatures:

$$
\beta_{i}=\frac{f_{i}(R)-f_{o}(R)+\kappa^{2} R^{2}}{2 \kappa R}, \quad \beta_{o}=\frac{f_{i}(R)-f_{o}(R)-\kappa^{2} R^{2}}{2 \kappa R}
$$

Note that $\beta_{\alpha}= \pm \sqrt{\dot{R}^{2}+f_{\alpha}(R)}$ automatically satisfy

$$
\beta_{i}-\beta_{o}=\kappa R
$$

Physically, the extrinsic curvature is positive (negative) if the outward pointed normal points toward larger (smaller) $r$. Hence, for a given trajectory of the domain wall (as given by $(2.5)$ and (2.6)), one can find the extrinsic curvatures $\beta_{\alpha}$, and thereby determine which types of bubble trajectories are compatible and which are inconsistent. This allows us to construct the appropriate Penrose diagram.

We are interested in geometries describing a bubble of de Sitter in Schwarzschild-AdS. So the inner and outer metrics can be written in static coordinates as (2.1), (2.2) with

$$
f_{i}(r)=1-\lambda r^{2}, \quad f_{o}(r)=1+r^{2}-\frac{\mu}{r}
$$

The three independent parameters $\lambda>0, \mu>0$ and $\kappa>0$ are related to the de Sitter cosmological constant, the mass of the black hole and the tension of the shell 8 . We will work in units where the AdS radius is one. Then the relevant length scales are the AdS radius, the de Sitter radius $r_{d}=1 / \sqrt{\lambda}$, and the black hole horizon radius $r_{+}$. The horizon radius $r_{+}$is defined by $f_{o}\left(r_{+}\right)=0$, so for large $\mu$ we have $r_{+} \sim \mu^{1 / 3}$. We will now discuss the domain wall solutions found in this case - a more general family of solutions is described in Appendix A.

7 In spacetimes with horizons, such as de Sitter or Schwarzschild-AdS, the static coordinates of the type used in (2.1), (2.2) are not globally well defined. So two distinct points in the spacetime can have the same value of $r, t$ and $\Omega$. Typically one can distinguish such points either by passing to a good global coordinate chart, such as the Kruskal coordinates for Schwarzschild-AdS, or by a further specification of the imaginary part of the time coordinate, $\Im(t)$.

8 More precisely, in terms of the actual cosmological constant $\Lambda$ and the ADM mass of the black hole $M, \lambda=\Lambda / 3$ and $\mu=2 G_{N} M$. 


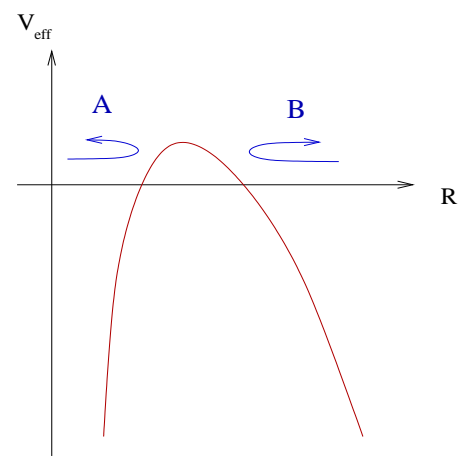

(a)

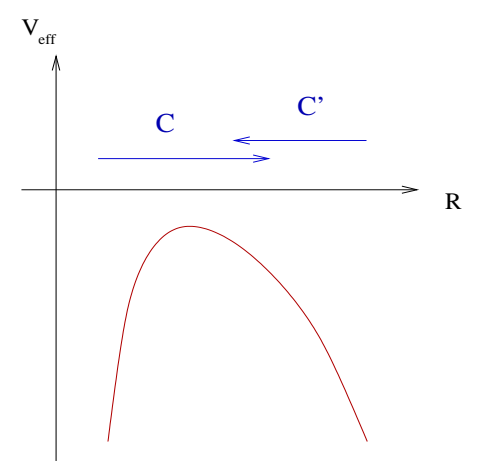

(b)

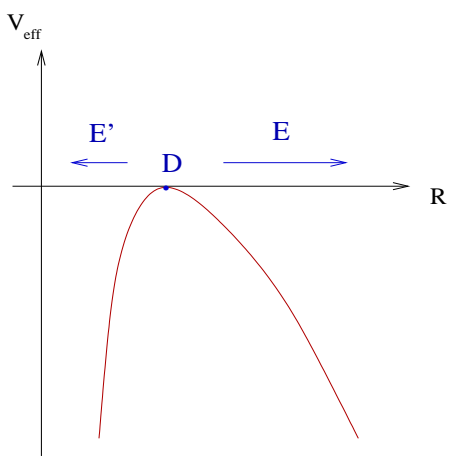

(c)

Fig. 2: Possible types of trajectories in an effective potential $(\overline{2.10})$, with $V_{\text {eff }}(r) \rightarrow$ $-\infty$ both as $r \rightarrow 0$ and as $r \rightarrow \infty$. (a): $V_{\max }>0$, (b): $V_{\max }<0$, and (c): $V_{\max }=0$. Trajectories $A, B$, and $D$ are time-symmetric (case $D$ describes a static shell), while the others are not time-symmetric.

Evaluating (2.6) for the specific case (2.9) gives the following effective potential:

$$
V_{\mathrm{eff}}(r)=-\left[\frac{\left(\lambda+\kappa^{2}-1\right)^{2}+4 \lambda}{4 \kappa^{2}}\right] r^{2}+1+\mu \frac{\left(1+\lambda-\kappa^{2}\right)}{2 \kappa^{2}} \frac{1}{r}-\frac{\mu^{2}}{4 \kappa^{2}} \frac{1}{r^{4}}
$$

The behavior of the shell can be read off from this effective potential. Both the $r^{2}$ and the $1 / r^{4}$ coefficients are negative, so $V_{\text {eff }} \rightarrow-\infty$ at $r \rightarrow 0$ and $r \rightarrow \infty$ and the potential has a maximum $V_{\max }$ at some value of $r$, say $r=r_{0}$. The possible domain wall trajectories $R(\tau)$ depend on the sign of $V_{\max }$ (recall that the effective 'energy' is zero), as indicated in Fig. 2. If $V_{\max }>0$, as sketched in Fig. 2a, there are two possible types of time symmetric situations: the shell can expand from zero size and recollapse (case A), or it can contract from infinite size and re-expand (case B). On the other hand, if $V_{\max }<0$ as in Fig. 2b, then no time symmetric situation exists: the shell either expands (case C) or contracts (case $\mathrm{C}^{\prime}$ ) on its semi-infinite trajectory. Finally, if $V_{\max }=0$ as in Fig. 2 $\mathrm{c}$, then we can consider a static shell sitting at $R(\tau)=r_{0}$ (case $\mathrm{D}$ ). Such a case requires a certain fine-tuning of the parameters to obtain $V_{\max }=0$, as well as of the initial conditions: $R\left(\tau_{0}\right)=r_{0}, \frac{d}{d \tau} R\left(\tau_{0}\right)=0$. If the latter is relaxed, the bubble may expand forever (case E) or collapse (case E'). Of course, the time reverse where the bubble slowly settles to $R(\tau \rightarrow \infty)=r_{0}$ is also possible.

We can now write down the Penrose diagrams for these various cases. Note that to do so we must take into account the sign of the extrinsic curvature as mentioned above. The details of this extrinsic curvature analysis are contained in Appendix A - we will simply quote the answers here. We will take the spacetime inside the bubble to be on the left of the wall trajectory in the Penrose diagram, and the outside spacetime on the right. 


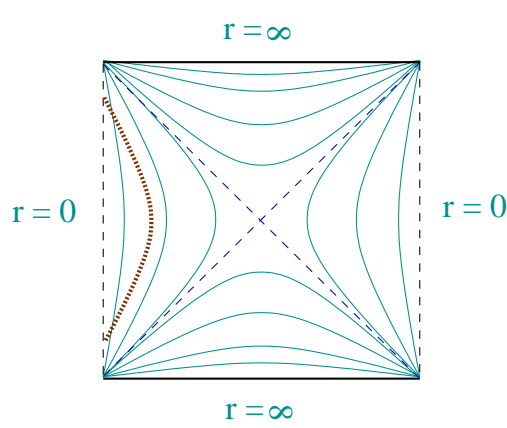

(a)

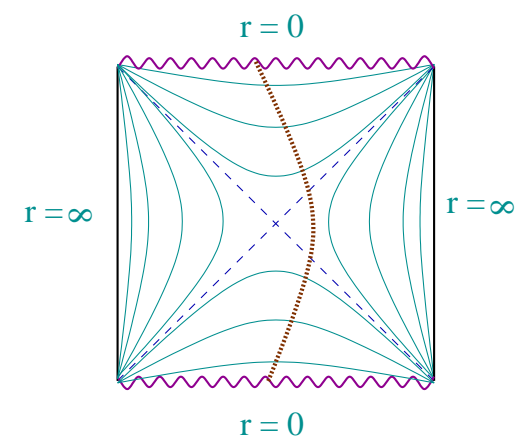

(b)

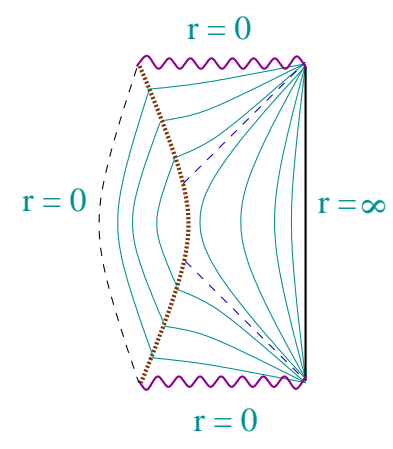

(c)

Fig. 3: Sketches of Penrose diagrams for (a) de Sitter, (b) Schwarzschild-AdS, and (c) de Sitter/Schwarzschild-AdS domain wall spacetimes, with constant- $r$ surfaces indicated. The dashed vertical lines correspond to the points $r=0$ in de Sitter, the dashed diagonal lines are horizons, the horizontal squiggly lines are the singularities, and the bold lines indicate the boundaries. The thick dotted lines indicate a possible trajectory of a shell across which the two spacetimes (a) and (b) may be patched together to obtain (c).

The Penrose diagrams 9 for de Sitter and Schwarzschild-AdS are given in Fig. 3a and Fig. $3 \mathrm{~b}$, along with constant- $r$ surfaces. In the de Sitter geometry, $r$ increases from 0 at the 'origin' (indicated by the dashed vertical lines in Fig. 3a), through the cosmological horizon $r=r_{d}$ (diagonal dashed lines), to $r=\infty$ at de Sitter $\mathcal{I}$ (bold horizontal lines). In Schwarzschild-AdS, on the other hand, $r=0$ at the singularities (indicated by the horizontal squiggly lines in Fig. 35b), increases through the black hole horizon $r=r_{+}$(diagonal dashed lines), and becomes infinite at the AdS boundary (bold vertical lines). A possible trajectory of the shell is further sketched on both spacetimes as a thick dotted curve. The corresponding junction spacetime is found by patching the two Penrose diagrams together along the shell, as shown in Fig. 3 3 c. Recall that the shell's trajectory must of course pass through the same values of $r$ on both sides, given by $r=R(\tau)$. This means that if the shell starts out from zero size, expands, and recontracts, its trajectory must correspondingly start and end on an origin of de Sitter, and on a singularity in Schwarzschild-AdS, as sketched. Note that in the resulting diagram (Fig. 3ic), $r=0$ on the left, top, and bottom of the diagram, and $r=\infty$ only on the right vertical line.

As is apparent from the Penrose diagrams, in a time symmetric set-up the shell reaches its maximum/minimum size $R_{t}$ at the $t=0$ slice (which passes horizontally through the

9 These Penrose diagrams, as well as the constant- $r$ surfaces, are merely sketches; in actuality, the singularity would be curved in, etc., as in [31] for Schwarzschild-AdS. Since they nevertheless capture many features of the causal structure, they are presented here and in the subsequent figures as sketches for ease of visualization. 
middle of the diagrams and forms a symmetry axis). In de Sitter, this size $R_{t}$ is necessarily bounded from above by the de Sitter radius $r_{d}$ (i.e., $R_{t} \leq r_{d}$ ), whereas in SchwarzschildAdS, $R_{t}$ is bounded from below by the black hole horizon $r_{+}$(so that $r_{+} \leq R_{t}$ ). We conclude that

$$
r_{+} \leq r_{d} \quad \text { for all time symmetric configurations. }
$$

It immediately follows that the black hole entropy is smaller than the de Sitter entropy for time-symmetric domain wall configurations; the implications of this surprising fact are discussed in the next section. However, we will see that there also exist time asymmetric solutions where the the black hole entropy is larger than the de Sitter entropy.
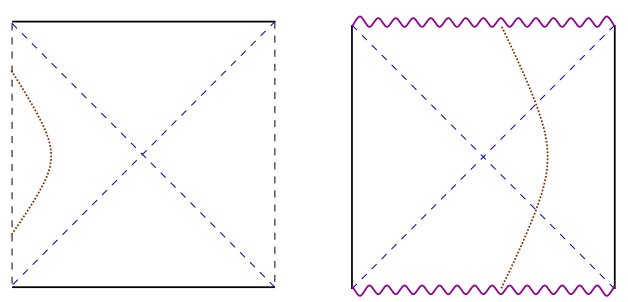

(A1)
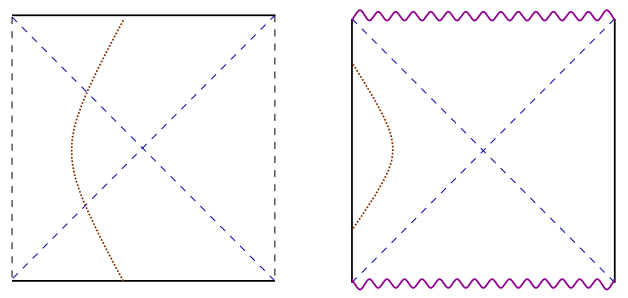

(B1)
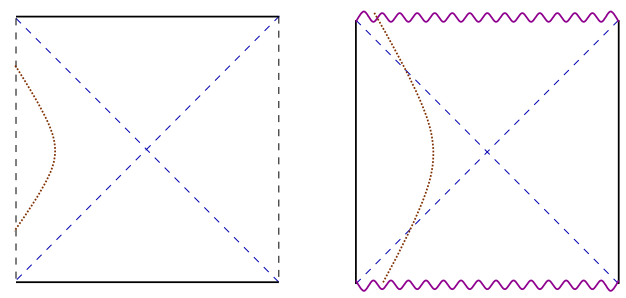

(A2)
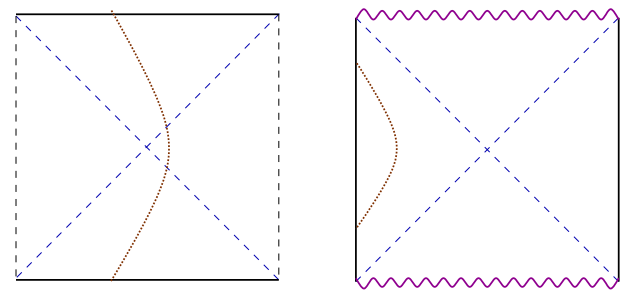

(B2)

Fig. 4: Shell trajectory corresponding to the time-symmetric cases (A) and (B) of Fig. 2, sketched on the de Sitter and Schwarzschild-AdS Penrose diagrams.

We will now examine in greater detail what types of trajectories (and corresponding Penrose diagrams) are admissible. The effective potentials for the different cases indicated in Fig. 2, along with distinct possibilities for the extrinsic curvatures, are plotted in Fig. 12 in Appendix A. The time symmetric trajectories, corresponding to the top two cases (A and B) drawn in Fig. 12, are depicted on the spacetime diagrams in Fig. 4. The distinguishing feature between $\mathrm{A} 1$ (B1) and A2 (B2) is the sign of $\beta_{o}\left(\beta_{i}\right)$ at the turning point; the former include fewer bifurcation points. Similarly, the time asymmetric trajectories, described by the cases (C) and (E) of Fig. 12, as well as the static case (D), are shown on the respective spacetimes in Fig. 5. Along with (C1) and (C2), which start from $r=0$ and expand forever, we could of course also have their time reverse, as indicated by case (C') of Fig. 2; 

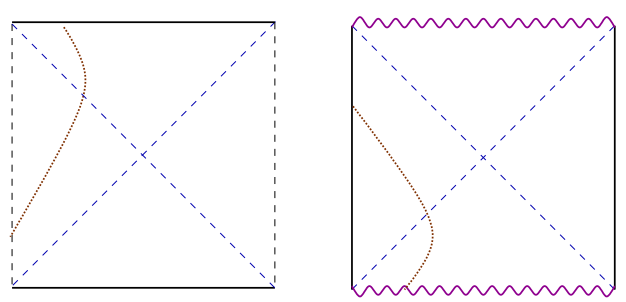

(C1)
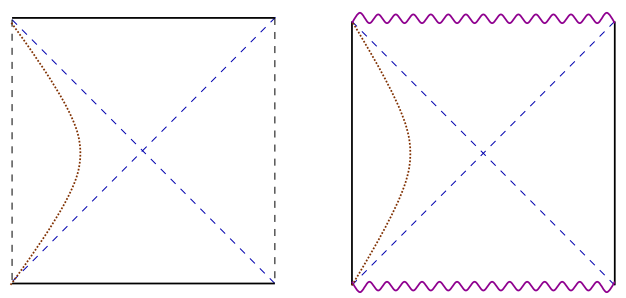

(D)
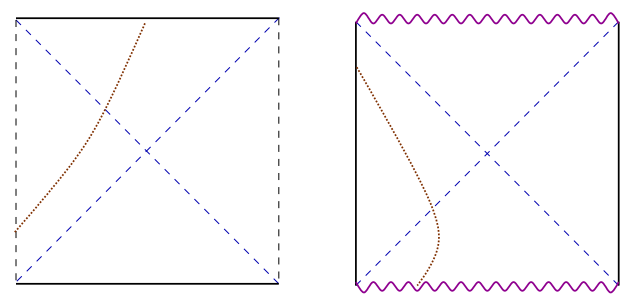

(C2)
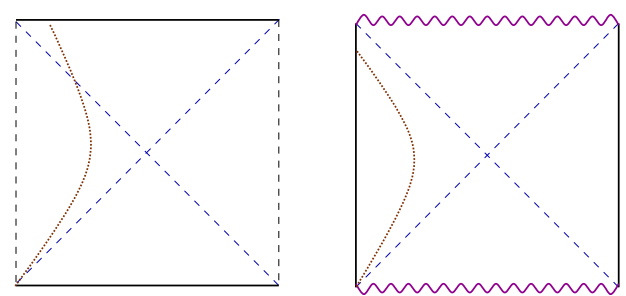

(E)

Fig. 5: Shell trajectory corresponding to cases (C), (D) and (E) of Fig. 2, sketched on the de Sitter and Schwarzschild-AdS Penrose diagrams. In all cases, we glue together the left spacetime and the right spacetime across the domain wall, discarding part of each diagram.

and similarly for case (E). As previously, the distinction between (C1) and (C2) comes from the sign of $\beta_{i}$ at large $r$. Case (D) is somewhat special: it corresponds to a static shell. Here we can have a global Killing field which is timelike everywhere outside the horizons. However, this geometry does not contain a piece of de Sitter $\mathcal{I}$.

We can now combine Penrose diagrams for the full junction de Sitter/SchwarzschildAdS spacetime, as in Fig. 3c. The result is sketched in Fig. 6.

Of the geometries described above, case $\mathrm{A}$ is an example of a false vacuum bubble that is excited in the true vacuum which re-collapses. These geometries are very similar to time symmetric spacetimes representing black hole collapse, except that the interior geometry is one with a different value of the cosmological constant. Cases B, C, E are the most interesting ones from our perspective, since here we see the presence of an inflating region of spacetime with de Sitter $\mathcal{I}^{+}$. These are the geometries we will be interested in describing holographically from the boundary field theory living on the AdS boundary on the right. A crucial feature in these geometries is that the inflating region is hidden behind a black hole horizon from the AdS boundary. We shall later show that this situation is generic as long as the matter fields making up the domain wall satisfy the null energy condition.

Note that in case $\mathrm{C}$, the de Sitter horizon is not necessarily larger than the 


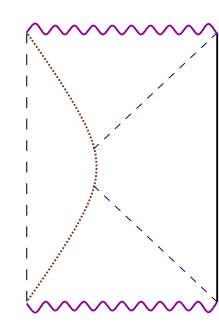

(A)

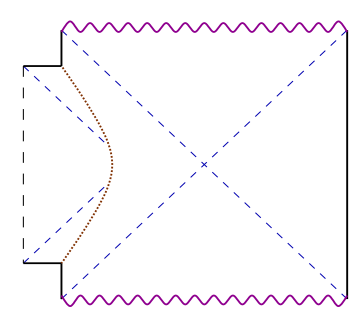

(B)

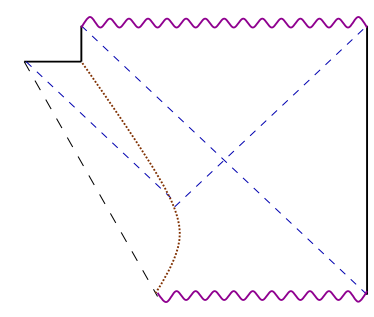

(C)

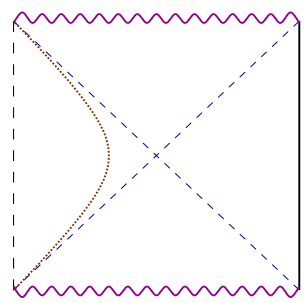

(D)

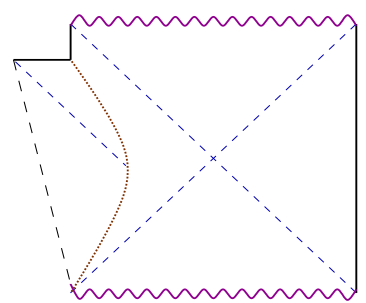

(E)

Fig. 6: Sketches of the full Penrose diagrams combined from the corresponding cases of Fig. 目 and Fig. 5. Metrically, the space on the left of the shell (thick dotted curve) is de Sitter, while the space on the right of the shell in Schwarzschild-AdS.

Schwarzschild-AdS horizon. In particular, which area is bigger depends on whether the de Sitter horizon (as drawn by the left diagonal dashed line in Fig. 6C) crosses the shell earlier or later than the black hole horizon. Since the radial coordinate increases monotonically along the shell, if the de Sitter horizon intersects the shell before the black hole horizon (at smaller $r$ and lower on the Penrose diagram), then $r_{d}<r_{+}$; conversely, if it intersects later, the de Sitter is bigger. Which of these is the case depends on the specific values of the parameters, but both possibilities are allowed 0 .

It is possible to start with pure AdS space and smoothly deform parameters to obtain a geometry with an inflating region. A sequence of spacetimes which illustrates this is shown in Fig. 7. We can deform from one spacetime to the next in Fig. 7 by smoothly adjusting the bulk initial data - in particular, the size of the false vacuum bubble - on the $t=0$ spacelike slice. Note that although the local geometry does not vary much in this progression, the global properties vary substantially from case to case. Namely, (a) is causally trivial; in (b) the spacetime has an event horizon; (c) acquires regions which are causally disconnected from the boundary; in (d) the shell itself passes through such a region; in (e) the entire shell is causally disconnected from the boundary; and finally

10 As explained in Appendix C, if in addition we require an initial Cauchy slice whose area increases monotonically and whose de Sitter part has domain of dependence which contains a piece of the de Sitter $\mathcal{I}$, then we necessarily obtain $r_{d}<r_{+}$. 


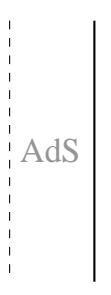

(a)

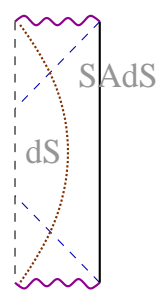

(b)

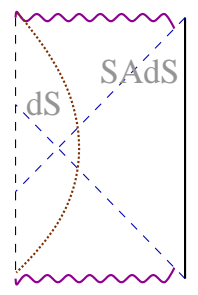

(c)

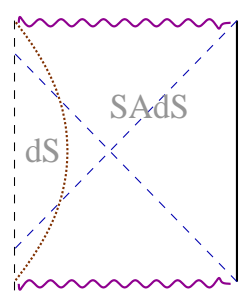

(d)

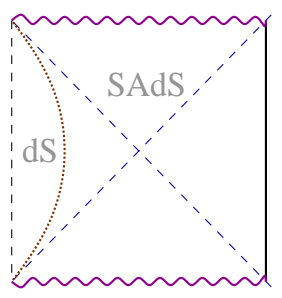

(e)

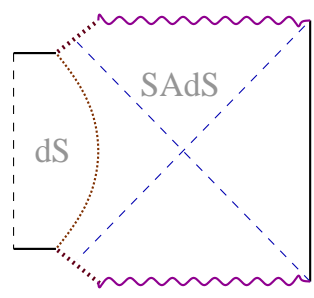

(f)

Fig. 7: Sketch of Penrose diagrams obtained by continuous deformations of the initial data and parameters, starting with pure AdS and ending with de Sitter (including the future and past boundary $\mathcal{I}^{ \pm}$) in Schwarzschild-AdS. Note that (b) is possible only for small black holes $r_{+}<r_{A}$, and in (f) the de Sitter $\mathcal{I}$ is generically joined to the Schwarzschild-AdS singularities by some metric which depends sensitively on the evolution (and hence drawn by a dotted line) but is unimportant for our discussion.

(f) acquires additional (de Sitter) asymptotic regions11. Naively, one might expect that if (a) is described by a holographic dual, then so will (f), since it seems unnatural that the holographic encoding would cease abruptly in this progression. However, as we argue later, the nature of the state may change.

\subsection{Beyond the thin wall approximation}

We have seen that in the thin domain approximation we can find geometries that contain both de Sitter $\mathcal{I}$ and AdS $\mathcal{I}$. Of course, we are really interested in the case of gravity coupled to a scalar field in a potential of the form sketched in Fig. 11. As described above, such potentials describe low energy dynamics on the string theory landscape. Moreover, the resulting spacetimes will be smooth. In Appendix B we will give a detailed argument that the basic features of the geometry do not change in this more general setup. In

11 For later discussions, we also note that running across the $t=0$ Cauchy slice of (d), (e) or (f) the size of spheres does not vary monotonically. In fact, for (e) and (f), the same is true for any Cauchy surface. 
particular, one can argue based on causality that there are solutions of scalar-gravity that contain both the de Sitter and $\operatorname{AdS} \mathcal{I}$. In this section we will discuss a few characteristic features of these solutions.

Perhaps the most important feature is the fact that the de Sitter $\mathcal{I}$ is causally disconnected from the AdS $\mathcal{I}$ - there is no null geodesic connecting the two. This is a very general property of any spacetime satisfying the null energy condition, including the scalar-gravity system under consideration. This can be seen from Raychaudhuri's equation for a congruence of null geodesics. Physically, because gravity is attractive, once a set of null geodesics start converging, they cannot diverge (unless they pass through an origin $r=0$ ). This immediately rules out null geodesics connecting the de Sitter and AdS $\mathcal{I}$ : a null congruence must converge to go into the bulk from an AdS $\mathcal{I}$, and diverge to reach the de Sitter $\mathcal{I}$ from the bulk.

Another curious property of some of the thin wall constructions is the presence of a part of AdS boundary on the left which disappears and appears as the shell attains infinite size. This second AdS boundary is an artifact of the thin wall approximation, and does not appear in the full solutions of scalar-gravity [35. Physically, any radiation emitted by the shell near the boundary would suffer a large blue-shift as it propagates into the bulk, and its backreaction would lead to a curvature singularity. This is somewhat analogous to the Cauchy horizon instability at the inner horizon of a charged or rotating black hole. While the exact nature of this singularity is of course difficult to determine due to its sensitivity to the initial domain wall profile, we expect that it will meet up with the Schwarzschild-AdS black hole singularity in a(n almost) null fashion, as sketched in Fig. 7ff. This can also be thought of as due to cosmic censorship 12 , because of an obstacle to Cauchy evolution (though by a boundary rather than a naked singularity). As is well known, asymptotically AdS spacetimes are not globally hyperbolic without specification of additional boundary conditions. Hence the appearance of an AdS boundary implies the formation of a Cauchy horizon for the evolution of the initial data as we cannot evolve the spacetime in the domain of influence of this boundary 13 . Now similarly, given generic perturbations on a Cauchy surface at finite time, we expect that a big bang singularity will remove any such AdS boundary in the far past. Hence it appears that these AdS boundaries are also artifacts.

So far we have limited our discussion to spherically symmetric geometries. However, there is a possibility that some of these geometries are dynamically unstable to aspherical fluctuations14. In particular, while the Schwarzschild-AdS and the de Sitter geometries are

12 We thank Gary Horowitz for pointing this out to us.

13 This is not an issue on the right AdS boundary, since there the CFT tells us what boundary conditions to impose.

14 We thank John McGreevy for alerting us to this possibility. 
individually dynamically stable to fluctuations, the shell itself might be unstable. If one considers the positions of individual pieces of the shell as determined by the effective potential $V_{\text {eff }}$, the shell's deformations will grow with time. This is because in the mechanical motion of particles away from the extremum of the effective potential (2.10) two particles tend to accelerate away from each other. A set-up similar to ours has been recently considered in [40], where it was shown that certain de Sitter/Schwarzschild-de Sitter domain walls are indeed unstable. Further it appears that changing the outside geometry there from Schwarzschild-de Sitter to Schwarzschild-AdS does not remove this instability [41]. It would be interesting to analyze this potential instability in detail for our set-up. We do not expect the instabilities to radically alter our story - for example, in the solutions with de Sitter $\mathcal{I}$, we expect the aspherical perturbations to remain small compared to the size of the bubble, as in [42,43]. Finally, we should emphasize that many of the cases considered above are fine-tuned, in the sense that they have been chosen to be time symmetric.

\subsection{A special parameter domain}

The arguments of the following sections will be sharpest for a certain range of parameters. This regime is given by $r_{d} \geq R_{t} \gg r_{+} \gg r_{A}=1 \gg \ell_{s}$ where, as a reminder, $\ell_{s}$ is the string length, $r_{d}=1 / \sqrt{\lambda}$ is the de Sitter radius of curvature, $r_{+}$is the black hole horizon

radius, which for large $\mu$ is given by $r_{+}=\mu^{1 / 3}, R_{t}$ is the domain wall position of the time symmetric solutions (cases A, B) at the turning point, and $r_{A}$ is the AdS curvature radius (which we have set to one). In this range of parameters the domain wall is very far away from the black hole horizon at all times, albeit causally disconnected from the right AdS boundary. Solving for the turning point of the effective potential (2.10), we find

$$
R_{t} \approx r_{+} /\left(1-\kappa^{2}\right)^{1 / 3}
$$

We see that to achieve the condition $R_{t} \gg r_{+}, \kappa$ must be close to one. Recall that $\kappa \sim \sigma r_{A} / m_{p}^{2}$ where $\sigma$ is the tension of the domain wall. A brief survey of known parts of the landscape reveals regions with $\kappa \gg 1$ and regions with $\kappa \ll 1$. There is no reason not to expect many vacua with $\kappa \sim 1$. Typically, if $\kappa \ll 1$ then $R_{t} \rightarrow r_{+}$. If $\kappa \gg 1$ then $R_{t} \rightarrow r_{+}$and $r_{+} \rightarrow 1 / \kappa$. Physically $\kappa \sim 1$ in AdS units is special because then the domain wall tension balances against the pressure which the true vacuum exerts on the domain wall. In flat space, such a balance would only be possible for one size of the bubble because the energy due to tension is proportional to the surface area of the domain wall, while the energy due to the pressure is proportional to the volume. In AdS, the two forces can almost cancel for a large range of bubble sizes because at scales big compared to the AdS radius, volume is proportional to surface area. For the special case of the static domain wall, we must also set the first derivative of the effective potential to zero, giving $\mu \sim 1 /\left(1-\kappa^{2}\right)^{2}$ and $R_{t} \sim 1 /\left(1-\kappa^{2}\right)$. 
The curvature at the maximum of the effective potential (2.10) goes to zero as $\kappa \rightarrow 1$ and $\lambda \rightarrow 0$. The instability growth rate of the static domain wall is governed by this curvature and hence goes to zero in this limit. As $\kappa \rightarrow 1$ the static domain wall becomes arbitrarily far away from the black hole horizon and its instability becomes arbitrarily small. Note that the relevant time scale for the instability is the Schwarzschild time of the asymptotic observer $t_{o}$ and not the proper time of the shell $\tau$. The conversion factor at large $R_{t}$ is $\tau \sim t_{o}\left(R_{t} / r_{A}\right)$. In a typical regime in parameter space, we have $t_{\text {instability }} \sim r_{d} r_{A} / R_{t}$, which can be taken to be large.

\section{Properties of the boundary CFT}

In the previous section we constructed a family of de Sitter/Schwarzschild-AdS domain wall spacetimes, shown in Fig. 6, some of which have inflating (de Sitter $\mathcal{I}$ ) regions. These solutions all have asymptotically AdS regions. Consider first the pure AdS geometry in Fig. 6 describing the stable ground state 15 of Fig. 11. If this is a vacuum of a consistent theory of quantum gravity (as we are assuming) then this theory defines a boundary CFT which represents the bulk via the AdS/CFT correspondence 16 . The scalar field representing the horizontal axis in Fig. 11 will be represented in the CFT and it seems plausible that one could excite a large number of its quanta to create the initial data for the de Sitter/Schwarzschild-AdS spacetimes17. However there is a puzzle about representing de Sitter degrees of freedom that at first glance casts doubt on this simple logic. This has to do with the fact that it naively appears that the boundary CFT must encode the dynamics of an enormous inflating region with far fewer active degrees of freedom accessible to it.

As we will see below, the resolution to this puzzle is that the boundary CFT is in a mixed rather than a pure state. Some mixed states arise by simply integrating out certain degrees of freedom in the CFT [44]. Conversely, certain pure states are expected to mimic mixed states to a high degree of accuracy [45,46]. We should emphasize that here we are claiming that the relevant mixed states arise because the boundary CFT is entangled with new degrees of freedom associated with de Sitter region behind the horizon. This is analogous to the appearance of the thermal density matrix in the standard eternal black hole [32, 33]. In fact, we will see that the present mixed states can be described in terms

15 To ensure stability, we may take this to be a supersymmetric minimum.

16 This CFT should in principle contain information about all accessible vacua in the landscape and hence will be an extraordinarily complicated object. We expect that the wide separation of tunneling time scales will enable us to focus on the truncated landscape of Fig. 1.

17 All geometrical scales can be taken much longer than string length and the coupling can be taken weak, so these gravity solutions should approximate the behavior of the full theory. 
of a cutoff Schwarzschild-AdS background - a fact which provides evidence that these spacetimes can be reliably described in AdS/CFT.

\subsection{The entropy puzzle}

The arguments presented above seem to imply that the field theory dual to the geometries which incorporate an inflating region is constructed by acting on the vacuum state with an appropriate set of local operators. This would lead us to conclude that the spacetime geometry is dual to the field theory in a particular pure state.

This picture however cannot be right, as it leads to a paradox regarding the entropy [34,35. Let us consider a de Sitter bubble with $\mathcal{I}^{+}$. As explained at (2.11), in the timesymmetric set-up, the size of the bubble on the $t=0$ slice, $R_{t}$, is smaller than the de Sitter radius and also larger than the black hole radius $r_{+}$. Hence we know that the black hole size $r_{+}$is necessarily smaller than the size of the de Sitter cosmological horizon $r_{d}$, implying that the black hole entropy is smaller than the entropy associated with the de Sitter false vacuum bubble.

The black hole entropy is associated with the number of active degrees of freedom in the boundary conformal field theory. This is the picture that is naturally suggested by black hole microstate counting using D-brane constructions. The de Sitter entropy, on the other hand, is a measure of the degrees of freedom necessary to define a quantum gravitational theory in de Sitter space [47]. Given this, it is hard to imagine how a pure state that is built out of the fewer black hole degrees of freedom can encapsulate the information required to describe the de Sitter space. This mismatch is what we term the entropy puzzle.

In fact, it is easy to see that this entropy mismatch can be made arbitrarily large after all, there is no restriction on the allowed de Sitter size at the level of classical geometries, since we could consider arbitrarily small positive values of cosmological constant. In a sense, we would have to use the vastly fewer degrees of freedom accessible to the boundary observer to describe the physics of arbitrarily many degrees of freedom. There exist (time asymmetric) geometries where the de Sitter entropy is less than the black hole entropy. We present the details of these solutions in Appendix C. Although the entropy puzzle is not present for these special cases, for time symmetric solutions the resolution lies in a different direction 18 .

We argued above that the cosmological solutions with asymptotically AdS regions should be described by a boundary CFT. However, the assumption that the geometry with an inflating region is described by a pure state of the CFT leads to an entropy puzzle. Since all of the solutions constructed in Section 2 were found by matching onto

18 For a different viewpoint on the entropy issue see 48 . 
Schwarzschild-AdS solutions, the AdS boundaries are separated from the inflating region by a black-hole horizon. Furthermore, as discussed earlier, this feature is guaranteed in any construction in classical general relativity with matter obeying the null energy condition. It is therefore natural to expect that the boundary CFT is very similar to the thermal field theory dual to a standard Schwarzschild-AdS black hole. A crucial feature of these spacetimes is that the conformal theory living on a boundary is in a mixed state, with density matrix

$$
\rho_{\beta}=e^{-\beta H}
$$

rather than a pure state 19 [49, 32, 33]. Here $H$ is the Hamiltonian of the CFT and $\beta$ the inverse temperature. In this section we will argue that the boundary CFTs dual to inflating geometries are also in a mixed state, whose density matrix differs from (3.1) only by small corrections.

In section 3.2 we will start with a discussion of mixed states in various extensions of Schwarzschild-AdS, before moving on to a more general discussion of mixed states and causal structure in section 3.3.

\subsection{Mixed states in asymptotically Schwarzschild-AdS geometries}

We will start with the most symmetric example of domain wall spacetimes, the static domain wall, shown in Fig. Te. In this geometry the domain wall is at a fixed radial position $R(\tau)=r_{0}=R_{t}$. Despite the fine tuning necessary to attain this geometry, it serves as a simple example to illustrate the general principle we wish to propose. In fact, as discussed in section 2.3 , by taking $\kappa \rightarrow 1$ we can dial $R_{t} \gg r_{+} \gg r_{A}$ and make the size of the instability vanishingly small. In the thin wall approximation, the spacetime to the right of the domain wall is identically Schwarzschild-AdS. If the location of the domain wall is far removed from the black hole horizon, $R_{t} \gg r_{+}$, we have a large region of spacetime where the usual picture of a Schwarzschild-AdS black hole should hold.

We will first consider the region of the spacetime in the Schwarzschild-AdS part of the geometry with $r \leq R_{t}$, i.e., imposing a cutoff at a radial scale $r_{c} \sim R_{t}$. To describe the physics of just this cutoff spacetime in the dual field theory, recall that for the usual eternal Schwarzschild-AdS black hole (see Fig. [3b) with $r_{+}>r_{A}$ the field theory dual is best described in the thermofield formulation. One associates a complete CFT Hilbert space to each AdS boundary of the black hole, labeled $\mathcal{H}_{L}$ and $\mathcal{H}_{R}$ respectively. These Hilbert spaces are non-interacting and the geometry is dual to a particular entangled pure state, the Hartle-Hawking state, in the tensor product Hilbert space $\mathcal{H}_{L} \otimes \mathcal{H}_{R}$ [36, 32, 33]. Tracing over one of the Hilbert spaces, say $\mathcal{H}_{L}$, leads to a self-contained description in $\mathcal{H}_{R}$,

19 Strictly speaking, this is true only for large black holes, with $r_{+}>r_{A}$. We will focus on this region of parameter space. 
but in a mixed state. The density matrix is the thermal density matrix (3.1) at the black hole temperature.

Physics in a cutoff Schwarzschild-AdS background is very similar to that of the noncutoff geometry. Now, however, the dual CFT is replaced by a conformal field theory cutoff at energy $E_{c} \sim r_{c} / r_{A}^{2}$. We denote the corresponding Hilbert spaces as $\mathcal{H}_{L, R}^{c}$. Concentrating on energy scales below $E_{c}$, we see that the entangled state description in $\mathcal{H}_{L}^{c} \otimes \mathcal{H}_{R}^{c}$ is still valid. So at low energies the right hand field theory will remain in a mixed state, which is now found by entangling $\mathcal{H}_{R}^{c}$ with the cutoff theory coupled to gravity. Of course, this procedure is ambiguous at scales near or above $E_{c}$, but at low energies the density matrix is given approximately by (3.1), with corrections that vanish as powers of $E / E_{c}$. Further, it is clear that the mixed state description is the correct one in $\mathcal{H}_{R} \supset \mathcal{H}_{R}^{c}$ so long as energy locality holds. This is a consequence of bulk locality (in $r$ ).

These arguments are best controlled when $R_{t} \gg r_{+}$obtained by taking $\kappa \rightarrow 1$. But as long as the domain wall is some macroscopic distance from the horizon, a macroscopic fraction of the excited degrees of freedom on the left should be entangled with those on the right, yielding a mixed state with macroscopic entropy of entanglement. The above arguments have been made in the $\mu>1$ "large" black hole regime. But it seems likely that even small black holes are described by entangled states and so these considerations should also apply to the $\mu<1$ regime as well.

Even at scales below the cutoff, the form of the density matrix is not unambiguously defined. In particular, the effective action of the cutoff CFT will include some number of irrelevant operators whose presence becomes important only at energies approaching $E_{c}$. Some of these effects can be calculated using bulk supergravity techniques.

We have not yet discussed the effect of the de Sitter region to the left of the domain wall. For all time symmetric collapse and static geometries the de Sitter radius $r_{d}$ must be larger than $R_{t}$. So we can treat the de Sitter region as a piece of essentially flat space. This results in significant modifications to the cutoff CFT because bulk massless propagators in flat space decay like powers of the proper distance between points, while in AdS space they decay exponentially. So bulk massless fields can (and do) induce nonlocal terms in the effective CFT. But we will show that these nonlocal effects can be made arbitrarily small by taking the cutoff surface defining the CFT much smaller than the domain wall.

To be specific take the horizon, cutoff and domain wall radii to have the following relative sizes: $R_{t} \gg r_{c} \gg r_{+}$. Take the most extreme case, a massless bulk field dual to a marginal operator $\mathcal{O}$ in the CFT. Ignoring the de Sitter contribution we have

$$
\left\langle\mathcal{O} \mathcal{O}^{\prime}\right\rangle \sim \frac{1}{r_{c}^{6} L^{6}} .
$$

Here $L$ is the geodesic distance between $\mathcal{O}$ and $\mathcal{O}^{\prime}$ on the cutoff surface. This formula follows from conformal invariance for marginal operators in a $D=3 \mathrm{CFT}$ or equivalently from 
summing over bulk particle paths in the AdS region to build up the massless propagator.

The de Sitter contribution behaves differently. Particle paths contributing to this behavior traverse a region of AdS space to the domain wall, then propagate in the de Sitter region, then re-enter the AdS region and return to the cutoff surface. We can calculate this contribution as the product of three propagators. Two account for the AdS propagation, each behaving like $\left(r_{c} / R_{t}\right)^{3}$. One accounts for the propagation through de Sitter space. This goes like $1 / x^{2}$, the standard massless particle propagator in $4 \mathrm{D}$ where $x$ is the distance between points. Folding these propagators together and integrating over joining points on the domain wall, we find that the massless propagation in the nearly flat de Sitter region induces a correction to the correlator that is long range compared to (3.2) (i.e., it falls off like a smaller power of $L$ )20. So when $r_{c} \sim R_{t}$ the de Sitter region makes a large nonlocal modification to the CFT. But if $R_{t} \gg r_{+}$we can choose $r_{A} \ll r_{c} \ll R_{t}$ in such a way that this correction is small compared to (3.2). So in this regime we can continue to make a controlled argument that the theory is in an entangled state.

While we have focused the discussion above on the case of the static domain wall, a similar situation can occur for any of the cases where the de Sitter bubble wall passes through the region to the left of the black hole, e.g., the cases b, c, d and e in Fig. 6. In particular consider Fig. 6]b, where the bubble expands in the far past and future. If the minimum bubble radius is much bigger than the radius of the black hole horizon, $R_{t} \gg r_{+}$, there is once again a large region to the right of the domain wall (and to the left of the horizon) where the geometry is Schwarzschild-AdS, and physics may be described in terms of a cutoff CFT. The discussion must be refined for the cases where the bubble shrinks towards either the future or past. However, it is clear that with some tuning, a large portion of Schwarzschild-AdS is relevant and a cutoff CFT can describe the physics for some large interval of time.

The arguments discussed above are our strongest evidence for the mixed state nature of the CFT description of inflation. They also provide the strongest evidence we have that these spacetimes can be represented in AdS/CFT.

To summarize, the CFT dual to geometries with an inflating false vacuum bubble is necessarily in a mixed state. In particular, this means that the active degrees of freedom in the boundary field theory, whose number is given by $\exp \left(S_{b h}\right)$, are entangled in a non-trivial way with the degrees of freedom in the inflating region (which, as we have argued before, could be much larger). In this picture, the black hole entropy $S_{b h}$ is simply a measure of this entanglement. The boundary observer who evaluates correlation functions in the

20 Essentially, modes which appear non-normalizable in the full Schwarzschild-AdS geometry become normalizable when the AdS boundary is "cut off" and replaced by the de Sitter bubble. This would have produced a massless graviton coupled the cut-off CFT [50,51] had we been considering the analogous constructions in higher dimensions. 
state dual to this geometry will conclude that the theory is in a mixed state with density matrix $\rho_{\text {bdy }}$ and an entanglement entropy $S_{b h}=S_{\text {ent }}=\operatorname{Tr}\left(\rho_{\text {bdy }} \log \rho_{\text {bdy }}\right)$. In our picture, the large number of de Sitter degrees of freedom are entangled with the black hole degrees of freedom. However, bulk locality suggests that they are entangled very weakly. So when these degrees of freedom are traced over, the resulting entanglement entropy $S_{\text {ent }}$ is much smaller than $S_{d S}$. Thus the mixed state picture avoids the entropy puzzle described above.

One striking aspect of this picture is the absence, say in the static domain wall case of Fig. 『e, of a second asymptotic boundary where the traced over degrees of freedom can be localized. This is in contrast to the eternal Schwarzschild-AdS black hole, where one traces over the degrees of freedom associated to one of the conformal boundaries. So it is natural to ask how one should describe the degrees of freedom that are entangled with the boundary CFT. One clue comes from the eternal Schwarzschild-AdS black hole, where bulk fields $\phi(r, t)$ in the right hand quadrant (see Fig. 9 b) can be moved to the left hand quadrant by shifting $t$ by half a Euclidean period, $-i \beta / 2$. Usually this transformation is used in the $r \rightarrow \infty$ limit where $\phi$ becomes an operator in the boundary CFT. This shift then relates the two boundary CFTs. But we can consider finite $r$ bulk fields as well. These can be constructed from the CFT fields by suitable coarse grainingen. For values of $r<R_{t}$, bulk fields in the left region, which can be described by the cutoff CFT, are related by this imaginary shift in $t$ to fields in the right region. So these degrees of freedom are accessible via analytic continuation. We will extend these considerations in Section 4.

The fact that a large number of degrees of freedom in the inflating region are entangled, albeit weakly, with CFT degrees of freedom allows us to use the latter to infer some properties of the former. We clearly cannot reconstruct all the information pertaining to inflation, but by virtue of the entangled state construction we have access to some of the information. Before proceeding to discuss how this information may be encoded in the boundary field theory, we turn to an interesting question: what are the situations in which the boundary theory is in a mixed state?

\subsection{Conditions for the appearance of mixed states}

We have argued that a broad class of de Sitter bubble solutions must correspond to mixed states in the CFT. However, we now wish to consider to what extent these arguments can be applied to other solutions, like the rapidly collapsing shells. A prori it is not clear whether the boundary CFT is in a mixed or a pure state. We will now proceed to discuss conditions which may delineate when a boundary CFT will be in a mixed state. We will consider more general situations than the cosmological solutions described above, and describe several possible scenarios under which mixed states arise. We will try to

21 See $52,53,54$ for examples of such coarse graining. 
formulate certain criteria for the appearance of mixed states; while some of these appear to be sufficient to guarantee a mixed state description, we are unable to determine which of these is necessary.

We will start by reviewing the bulk 22 explanation for the appearance of mixed states. According to the AdS/CFT correspondence, correlators of local operators in boundary field theories are found by taking bulk correlation functions to the boundary and stripping off the appropriate powers of radial coordinate. When there are regions in the spacetime that are causally disconnected from the boundary - i.e., regions that are outside both the past and future light cones of the boundary - then typically these bulk correlation functions are evaluated in a mixed state.

To see this, consider a quantum field $\phi$ in an asymptotically AdS spacetime with a moment of time symmetry $t \rightarrow-t$. The Hilbert space of this scalar field can be written in a position space basis at time $t=0$. When the spacetime contains a region causally disconnected from the boundary, the bulk Hilbert space can be factorized into two pieces $\mathcal{H}^{b}=\mathcal{H}_{R}^{b} \otimes \mathcal{H}_{L}^{b}$, where $\mathcal{H}_{R}^{b}$ is spanned by operators located inside the causal wedge of the boundary and $\mathcal{H}_{L}^{b}$ is spanned by operators in the causally disconnected region. To calculate the expectation value of local boundary operators, we only need to calculate bulk operators inside the light cone of the boundary. These operators act trivially on $\mathcal{H}_{L}^{b}$, so they are evaluated in the mixed state found by tracing over $\mathcal{H}_{L}^{b}$ :

$$
\rho_{R}^{b}=\operatorname{Tr}_{\mathcal{H}_{L}^{b}}\left|\psi^{b}\right\rangle\left\langle\psi^{b}\right| .
$$

Here $\left|\psi^{b}\right\rangle$ denotes the (pure) state of the quantum field $\phi$. A priori, $\left|\psi^{b}\right\rangle$ might be of the form $\left|\psi_{L}^{b}\right\rangle \otimes\left|\psi_{R}^{b}\right\rangle \in \mathcal{H}_{L}^{b} \otimes \mathcal{H}_{R}^{b}$, in which case $\rho_{R}^{b}$ has zero entropy and describes a pure state. However, one can show that if this is the case then quantum backreaction will be large near the horizon, destroying the spacetime. This is a familiar fact for black hole or Rindler horizons (see e.g., [55]). In black hole geometries the Boulware vacuum factorizes, and leads to a divergent stress tensor at the horizon. The same is true for the Rindler vacuum of an accelerating observer. More generally, if the state $\left|\psi^{b}\right\rangle$ factorizes then the expectation value of a set of local operators jumps discontinuously as one of the operators moves across the bifurcation point. In particular, such a correlation function vanishes unless all of the operators are located on the same side of the bifurcation point. So the value of the stress tensor, which can be found by differentiating a two point function, will typically diverge. We conclude that the density matrix (3.3) describes a genuine mixed state with non-zero entropy.

To summarize, if the standard AdS/CFT bulk to boundary dictionary is assumed in spacetimes with causally disconnected regions, we arrive at the following criterion 23

22 We will distinguish bulk quantum field theory data by an explicit $b$ superscript.

23 See Appendix D for a critique of this criterion. 
Criterion 1: Correlators of local operators in a boundary CFT are evaluated in a mixed state if there exist regions of spacetime that are causally disconnected from the boundary.

We should note that although the description in terms of a single boundary CFT is as mixed state, there may be additional descriptions of the geometry in terms of a pure state. For example, in the eternal Schwarzschild-AdS geometry discussed above the spacetime is described by two boundary CFTs in a particular pure entangled state. It is only by tracing out degrees of freedom on one side that one obtains the mixed state description of correlators on the other boundary 24 . We should emphasize that the description of the Schwarzschild-AdS geometry as a thermal state with density matrix $\rho_{\beta}$ on the right boundary only determines bulk correlators in the causal wedge of the right boundary. It does not, for example, unambiguously fix correlators of operators near the left boundary or correlators between operators in the left and right causal regions. This is because there are many choices of pure state $|\psi\rangle$ in the boundary Hilbert space $\mathcal{H}=\mathcal{H}_{L} \otimes \mathcal{H}_{R}$ which lead to the same density matrix $\rho_{R}$ upon tracing over $\mathcal{H}_{L}$. To describe the entire geometry, one needs to specify the pure state $|\psi\rangle$; typically it is specified by the Euclidean path integral with appropriate boundary conditions. It is only once one specifies $|\psi\rangle$ that one can, using, e.g., analytic properties, relate correlators in the entire spacetime to those in a single boundary CFT.

In more general spacetimes, such as the inflating geometries described above, it is not clear how to describe the mixed boundary state as a pure entangled state of a larger theory (or indeed whether such a pure state description exists). Given the fact that mixed state correlators unambiguously fix correlators only in the causal region, it is necessary to make an additional assumption in order to extract behind the horizon physics. As we will describe in the next section, we will typically assume analyticity in the gravity description, which in many cases amounts to defining a bulk state $\left|\psi^{b}\right\rangle$ on a complete Cauchy surface by Euclidean continuation. This leaves as implicit the construction of a pure entangled state in the dual holographic theory.

One may wish to conjecture a stronger criterion where "if" is replaced by "if and only if". However, 44] considers a mixed state described by pure AdS space but where the foliation lends itself to tracing over the CFT degrees of freedom on half of the boundary. Certainly here there are no regions causally disconnected from the full boundary. Rather

24 There is reason to expect that this behavior is a general feature of spacetimes with multiple asymptotic AdS boundaries. In general, solutions to Einstein's equations contain multiple asymptotic AdS boundaries only under very specific circumstances [56,57,58]. Typically, such solutions have singularities in both the far past and the far future and the conformal boundaries are causally disconnected. Thus the boundary CFTs do not interact, but are evaluated in an entangled state of the form described here. 
it is only that degrees of freedom on different components of the same boundary are entangled. Hence a stronger conjecture might be made as

Criterion 1': Correlators of local operators in a boundary CFT are evaluated in a mixed state if and only if there exist regions of spacetime that are causally disconnected from the corresponding boundary components.

One drawback of either of the criteria outlined above is that the presence of a causally disconnected region is a global property of the spacetime. In AdS/CFT, one expects bulk Cauchy evolution to correspond to Hamiltonian evolution in the boundary. One is therefore tempted to conclude that the prescription of Cauchy data on a spacelike slice suffices to determine the state of the boundary theory. This criterion depends only on the behavior in the neighborhood of a spacelike slice, and only very indirectly on global properties of the spacetime. It should not be necessary to evolve the data, and then infer from this bulk evolution that the spacetime has a causally disconnected region, to conclude that dual CFT state is mixed. Furthermore, there appear to be explicit examples with causally disconnected regions which are nevertheless described by pure boundary states - these are discussed in Appendix D.

We have been careful to focus the above discussion on local operators, whose correlation functions are easily extracted from bulk correlators. However, there are other possible criteria for the existence of mixed states which do not rely on bulk field theory arguments. For example one can motivate a criterion based on the scales the boundary observer can probe, which we expect by ideas of holographic renormalization to be related to the proper size of the spheres in geometries with spherical symmetry. Consider a spacelike slice (say along $t=0$ ) with metric

$$
d s_{t=0}^{2}=d r^{2}+R(r)^{2} d \Omega^{2}
$$

Geodesics with angular momentum $L$ in the geometry (3.4) achieve a minimum radial scale, $R_{\text {min }}=L$, before turning back. If we consider correlation functions of high dimension operators at fixed large $r$, the two point function is given by the geodesic length 25 . Since the geodesic turns around it only samples part of the geometry (3.4). Further, however, the variation of the corresponding correlator with $L$ is a sharp probe sampling the geometry at $R_{\text {min }}$ and implicitly the CFT at the corresponding energy scale. If $R(r)$ were monotonic, in the limit $L \rightarrow 0$ we can probe any radial interval down to $R(r)=0$. In contrast, if $R(r)$ is not monotonic, there will be intervals which this probe cannot access. This restriction on the scales that can be probed by the boundary correlators is evocative of the c-theorem,

25 Strictly speaking, these geodesics appear as local extrema of a path integral, and an additional calculation is needed to determine where they are the dominant contribution. We'll ignore this subtlety for now. 
if we assume that the size of the spheres is a sensible measure of the effective number of degrees of freedom. This is suggestive then that whenever we have a Cauchy slice with a non-monotonic proper size for the spheres (as for example in the eternal Schwarzschild-AdS black hole) there are degrees of freedom that are not accessible to the boundary observer. One can therefore conjecture an alternate criterion for a mixed state description in the boundary field theory:

Criterion 2: When the spacetime has spherical symmetry, boundary correlators are evaluated in a mixed state only when the radial sizes of the spheres are non-monotonic along the spacelike slice at $t=0$.

We should emphasize that this differs from the first criterion described above. In particular, there are spacetimes where $R(r)$ is monotonic, but nevertheless the regions of small $R$ are causally disconnected. An example is a collapse spacetime, where a shell of matter is sent in from asymptotic infinity to create a black hole in the interior, as discussed in Appendix D. Another distinction is that this criterion is local in time - Cauchy data alone suffices to determine whether or not a state is pure. Of course, implicitly here we are only considering time symmetric configurations. As discussed in Appendix $\mathrm{C}$, there are more general solutions where Cauchy slices may be chosen to have the proper sizes of spheres varying either monotonically or non-monotonically. It is far from clear how to extend this criterion to such cases.

This criterion was derived from holographic considerations and relies strongly on the choice of spacelike slices. One might therefore attempt to formulate a more covariant criterion, in terms of null slices rather than spatial slices. Demanding that the sizes of spheres be monotonic along lightlike rather than spacelike directions is equivalent to the condition that there be no additional holographic screens, as defined by Bousso [59]. It is straightforward to construct asymptotically AdS spacetimes for which this criterion differs from the other two, so for completeness we summarize this as

Criterion 3: The correlators of operators in a boundary CFT are evaluated in a mixed state only if the spacetime has additional holographic screens.

The criterion conjectured here should probably be refined as one finds additional holographic screens (beyond the AdS boundary) for any collapsing bubbles (even Fig. [7b). Naively, at least, very light bubbles such as this would be in a pure state. Of course, this just underscores our problem that holography in general spacetimes remains poorly understood.

We have not undertaken an extensive classification of the differences between these three criteria. It is however easy to construct examples where we obtain differing results depending on the criterion chosen. For example, in the progression sketched in Fig. 7, 
Criterion 3 applies to cases (b) - (f), Criterion 1 to cases (c) - (f) and Criterion 2 to cases (d) - (f). As should be apparent, we are unable to offer a single criterion that is both necessary and sufficient for the CFT to be in a mixed state. This remains an interesting open problem.

\section{Probes of inflation in AdS/CFT}

In the preceding sections we have shown that it is possible to construct, within the classical approximation, domain wall spacetimes that interpolate between de Sitter and AdS. In some cases, one can obtain a large inflating region, which is separated from an asymptotic AdS boundary by a black hole horizon. We have argued that in these cases the dual boundary theory is in a mixed state. In this section we will discuss the extent to which boundary CFT observables, calculated in this mixed state, contain information about the inflating region.

At first glance, we might worry that the mixed state results from tracing over the degrees of freedom behind the horizon, including the ones describing inflation, and so no vestige of these degrees of freedom will be visible. This is not the case 2 . In fact, the traced-over degrees of freedom leave a substantial imprint on the mixed state which can be analyzed. One well known example of this is the eternal Schwarzschild-AdS black hole where correlators in the thermal density matrix describe the degrees of freedom on the right AdS boundary. Analytically continuing the arguments of operators in imaginary time gives correlators describing degrees of freedom on the left boundary27. In the following section we will use analyticity to probe degrees of freedom in the inflating region. We expect correlators to be analytic for a wide variety of physically interesting states, including those under discussion here.

In section 4.1 we will follow the strategy of [29,30,31] to probe physics behind the horizon, and examine correlation functions of high dimension operators in the boundary theory. For such operators, a boundary two point function is given by the length of the bulk spacelike geodesic which connects the two points. We will show that the presence

26 Heuristically, this can be seen by considering an entangled state in a tensor product of two identical Hilbert spaces, each of dimension $d, d \gg 1$. While an entangled pure state in the product space is described by $d^{2}$ complex parameters, The density matrix obtained by tracing over one Hilbert space is described by $d^{2} / 2$ complex parameters. Roughly speaking, the density matrix can pin down "half" of the entangled state. Unitary transformations of the traced over space leave the density matrix unchanged and account for hidden information. There are only $d$ independent pure states in each individual Hilbert space. The density matrix carries far more information than the selection of a pure state.

27 For reviews of these analyticity concepts see, for example, [28, 30, 31] 
of de Sitter $\mathcal{I}^{+}$leads to a singularity in the correlation functions of the boundary theory, after appropriate analytic continuation. In section 4.2 we will describe an even more dramatic probe of the inflating region. In the classical approximation, the boundary CFT can be analytically continued to construct dS/CFT correlators living on the asymptotic de Sitter $\mathcal{I}^{ \pm}$. Once quantum effects are included this provides a powerful probe of the non-perturbative physics of the inflating region.

\subsection{Geodesics probes of domain wall spacetimes}

We will start by considering correlation functions of boundary operators $\mathcal{O}(x)$ with large dimension $\Delta$ describing bulk particles with mass $m \sim \Delta$. In the limit where $m$ is large the two point function can be evaluated in semiclassical approximation and is given by

$$
\langle\mathcal{O}(x) \mathcal{O}(y)\rangle \sim e^{-m \mathcal{L}(x, y)}
$$

where $\mathcal{L}(x, y)$ is the proper length of the spacelike geodesic connecting the two points on the boundary 28 . This length is formally infinite, so $\mathcal{L}$ is regularized by taking $x$ and $y$ slightly away from the boundary. In this section we will focus on radial geodesics, whose form can be found by patching together geodesics on either side of the domain wall along with an appropriate junction condition across the wall.

For radial geodesics in spacetimes of the form (2.1), the geodesic equations are

$$
\frac{d t_{\alpha}}{d \tau_{g}}=\frac{E_{\alpha}}{f_{\alpha}(r)}, \quad\left(\frac{d r}{d \tau_{g}}\right)^{2}=f_{\alpha}(r)+E_{\alpha}^{2},
$$

where $\alpha=i, o$ and $\tau_{g}$ is the affine parameter along the geodesic. $E_{\alpha}$ is a conserved quantity associated with the Killing vectors $\left(\frac{\partial}{\partial t_{\alpha}}\right)^{a}$ on either side of the domain wall. To trace geodesics through the junction, we assume that the domain wall is transparent i.e., an observer on the wall measures the same energy and momentum for a geodesic on both sides. We also use that $r$ is continuous across the junction. On the other hand, $t$ jumps discontinuously across the domain wall. We can determine the jump in $t$ from the normalization of the 4 -velocity $u^{a}$ of the shell:

$$
u^{a} u_{a}=-1=-f_{\alpha}(r) \dot{t}_{\alpha}^{2}+\frac{\dot{r}^{2}}{f_{\alpha}(r)} .
$$

28 There are a few subtleties in this argument, which we will mostly neglect in the following. In particular, one must prove that the geodesics under consideration lie on the path of steepest descent in order to contribute to the correlator. Even if this is not the case, however, the effect described below will still be visible upon analytic continuation of correlation functions. To track the "metastable" geodesic reliably requires $m \rightarrow \infty$. The heaviest particles available as probes are wrapped D-branes with masses of order $1 / g_{s}$. So this technique requires the ability to take $g_{s} \rightarrow 0$. There are examples in the landscape [11] where this is possible. 
Using $\dot{r}^{2}=-V_{\text {eff }}(r)$ gives

$$
t_{\alpha}(r)=-\int \frac{\beta_{\alpha}(r)}{f_{\alpha}(r) \sqrt{-V_{\text {eff }}(r)}} d r .
$$

To find the explicit relation between $t_{i}$ and $t_{o}$ we would have to invert (4.4). Moreover, to determine where a particular spacelike geodesic intersects the bubble trajectory, we would have to solve the implicit equations $R(\tau)=r\left(\tau_{g}\right)$ and $t_{\text {bubble }}(\tau)=t_{\alpha}\left(\tau_{g}\right)$. Finally, to obtain $t_{\alpha}\left(\tau_{g}\right)$ and $r\left(\tau_{g}\right)$, we must invert the expressions for $\tau_{g}(t)$ and $\tau_{g}(r)$ from (4.2). Therefore, except in very special cases, one can not write a closed form expression for the geodesic.

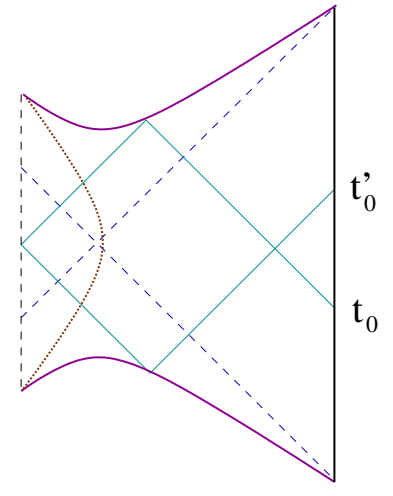

(a)

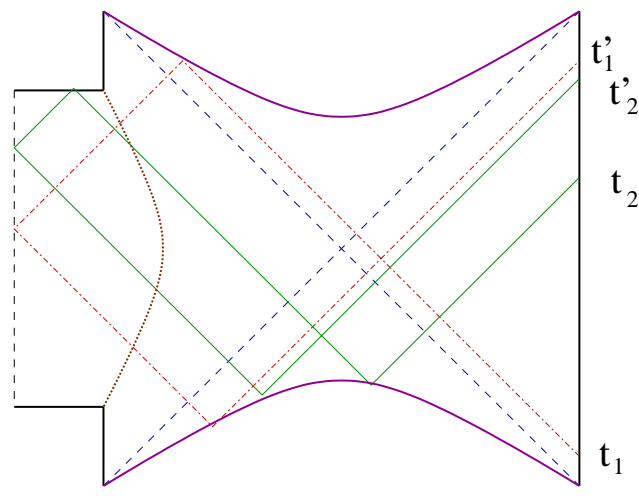

(b)

Fig. 8: A few null geodesics in thin domain wall spacetimes. (a) Collapsing false vacuum bubble geometry (b) Inflating false vacuum bubble geometry.

For certain special geodesics, $\mathcal{L}$ vanishes - i.e., the geodesic becomes null - and the two point function $\langle\mathcal{O}(x) \mathcal{O}(y)\rangle$ has a singularity. Such null geodesics are found by taking the $E \rightarrow \infty$ limit of the spacelike geodesics described above. In this case it is simple to solve for the geodesics explicitly. The Penrose diagrams for two domain wall geometries, along with a few null geodesics, are sketched in Fig. 8. While the black hole singularities are drawn curved [31], on a true Penrose diagram, de Sitter origin and $\mathcal{I}^{ \pm}$would also bend.

The null geodesics Fig. 8 are drawn as bouncing off the black hole singularity and de Sitter $\mathcal{I}$. This is because our null geodesics arise as a limit of spacelike geodesics, which are repelled from both the singularity and de Sitter $\mathcal{I}$. To see this, note that near the $r=0$ singularity of Schwarzschild-AdS, the geodesic equations (4.2) become

$$
\left(\frac{d r}{d \tau_{g}}\right)^{2}=E^{2}-\frac{\mu}{r} \Longrightarrow \frac{d r}{d \tau_{g}}=0 \text { and } \frac{d^{2} r}{d \tau_{g}^{2}}>0 \text { at } r=\frac{\mu}{E^{2}}
$$


We conclude that a spacelike geodesic is repelled by the singularity at a distance $r_{\min }=\frac{\mu}{E^{2}}$. Likewise, near the de Sitter $\mathcal{I}^{+}$the geodesic equation becomes

$$
\left(\frac{d r}{d \tau_{g}}\right)^{2}=E^{2}-\frac{r^{2}}{r_{d}^{2}} \Longrightarrow \frac{d r}{d \tau_{g}}=0 \text { and } \frac{d^{2} r}{d \tau_{g}^{2}}<0 \text { at } r=E r_{d}
$$

So a spacelike geodesic turns around at maximum radius $r_{\max }=E r_{d}$. This property, that spacelike geodesics are repelled by de Sitter $\mathcal{I}^{ \pm}$, is analogous to the fact that timelike geodesics are repelled by the timelike boundary of AdS. In the limit where the geodesic becomes null, $E \rightarrow \infty$, the geodesics simply bounce off the singularity and de Sitter $\mathcal{I}$.

We conclude that CFT correlators will have additional singularities due to null geodesics behind the horizon, of the form

$$
\langle\mathcal{O}(t, \Omega) \mathcal{O}(s,-\Omega)\rangle \sim \frac{1}{\left(s-t^{\prime}(t)\right)^{2 m}}
$$

where $t^{\prime}(t)$ indicates the point where the null geodesic starting at $t$ re-emerges on the boundary and depends on the particulars of the geometry. The operators are at antipodal points on the sphere because any geodesic that returns back to the AdS boundary has to pass through the origin of de Sitter, where it will move to the opposite side of the sphere.

Note that the singularities (4.7) seen in the analytically continued correlators are not time translation invariant, revealing the presence of interesting dynamics in the left causal region. In particular, these correlators give a clear signal of inflating bubbles. For example, inflating geometries like Fig. 8b give rise to two classes of null geodesics: those associated with the singularity at $t_{2}^{\prime}\left(t_{2}\right)$, which reflect off the de Sitter $\mathcal{I}^{+}$, and those associated with $t_{1}^{\prime}\left(t_{1}\right)$, which miss the $\mathrm{dS} \mathcal{I}^{+}$and bounce off the black hole singularity. So if one calculates the value of $t^{\prime}$ as a function of $t$, one finds a discontinuity as the corresponding geodesic passes the junction of the de Sitter and AdS boundaries. So the singularity (4.7) will disappear for a certain range of $t^{\prime}$. Moreover, we argued earlier that once back-reaction is included this junction is replaced by a big crunch singularity. These singularities do not repel geodesics, so the correlators will display an even more pronounced discontinuity. This behavior will not be found in the analytically continued correlators corresponding to non-inflating geometries like Fig. 8a, so provides a distinct signature of inflation in the boundary CFT.

\subsection{From AdS/CFT to $d S / C F T$ and beyond}

There are even more powerful probes of the inflating region available in this system, which build on analyticity. 


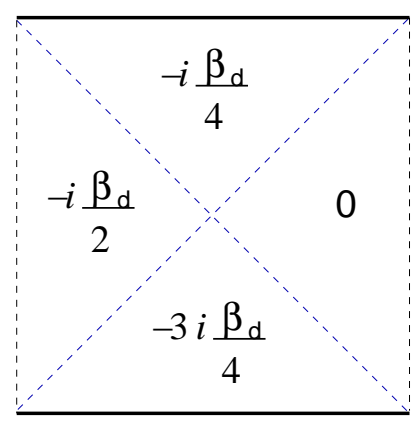

(a)

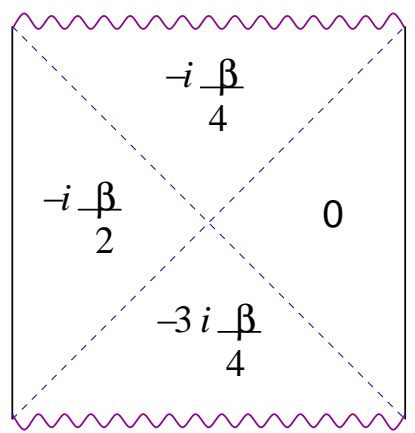

(b)

Fig. 9: Conventions for imaginary part of the time coordinate. (a) de Sitter spacetime (b) Schwarzschild-AdS geometry.

We start by recalling a basic manipulation in the eternal Schwarzschild-AdS black hole mentioned above. Schwarzschild-AdS can be described by four static coordinate patches, whose time coordinates have different imaginary parts. In our conventions, the imaginary part of the time coordinate is shown in Fig. 9b. For example, in the Schwarzschild-AdS geometry each time we cross a horizon going counter-clockwise on the Penrose diagram, we pick up a imaginary part $-\frac{i}{4} \beta$, where $\beta$ is the inverse Hawking temperature. So if we continue time by half a Euclidean period $\beta, t \rightarrow t-i \beta / 2$, we take a point on the right boundary of Schwarzschild-AdS to one on the left boundary.

In the domain wall spacetimes, however, there is no left boundary. So, instead we can consider a point at large but finite $r$. Now continue time by half a period. In the thin wall approximation the metric is just Schwarzschild-AdS (assuming $r$ is not too large). So this continuation yields a point in the left quadrant at the same $r$ and $\Re(t)$. Now one can move into the de Sitter region by continuing in (real) $r$. We here go beyond the thin wall approximation and assume, as is plausible, that the domain wall is smooth. There will be a sharp signal in a correlator as one of its points crosses the domain wall. (For concreteness imagine a two point function where both points have been continued to near the domain wall.) This will be a first sheet effect, visible at finite $g_{s}$, unlike the signal due to subdominant geodesics discussed above. It is not impossible for a two point function that is smooth on the AdS boundary to display a sharp signal under analytic continuation. As an example imagine eternal Schwarzschild-AdS with an operator inserted on the left boundary and the two correlated points on the right boundary. On continuation by half a period the correlated points can collide with the left hand operator giving a large signal. In fact, at finite $r$ the signal will be nonsingular, but as we move to the boundary $r \rightarrow \infty$ a domain wall of fixed bulk width becomes sharper and sharper in boundary variables. 
The boundary correlator will be singular, and possibly non-analytic. This is somewhat analogous to the Janus solution of [60,61].

Once we have changed $r$ enough to enter the de Sitter region, we may cross the de Sitter horizon by shifting the imaginary part of $t$ once more. Again, this is because in static coordinates de Sitter is covered by four coordinate patches, whose time coordinates have different imaginary parts - see Fig. 9a. So we may move through the de Sitter horizon by taking $t \rightarrow t-i \beta / 2-i \beta_{d} / 4$, where $\beta_{d}$ is the inverse de Sitter temperature. We should mention that smoothing out the domain wall to a small but finite thickness will change these Euclidean shifts by a small amount. Because the metric has bounded first derivative, these changes are uniformly small. Thus going beyond the thin wall approximation will not alter the basic picture we are describing.

Having moved points past the de Sitter horizon we can now study behavior near de Sitter future infinity by taking $r \rightarrow \infty$. If we consider a bulk scalar field on this classical geometry we know that correlators near de Sitter future infinity give the conformally invariant results of dS/CFT [19]. But correlators near the AdS boundary give AdS/CFT results. We thus see that analytic continuation takes AdS/CFT correlators into dS/CFT correlators. We will work out an explicit example of this in Appendix E, for the spacetimes described by Coleman and de Luccia 62]. In this case one can write down explicit formulas for boundary correlators that continue from AdS/CFT to dS/CFT. In this particular example, however, the AdS boundary will typically be destroyed by backreaction. So the explicit calculation in Appendix E may be thought of as a toy model for the full analytic continuation required to go from a Schwarzschild-AdS boundary to the de Sitter boundary.

We should emphasize that we can still implement the analytic continuation described above once quantum effects are taken into account. We have a non-perturbative definition of quantum gravity given by the full AdS/CFT system, so analytic continuation of its correlators gives a precise quantum gravity description of correlators near de Sitter future infinity. These correlators provide the non-perturbative completion of dS/CFT. In principle, this should include effects like bubble nucleation of other "pocket universes" in the far future 63]. Bubble nucleation is an $\exp \left(-1 / g_{s}^{2}\right)$ effect and so should be related to some instanton effect in the CFT, after appropriate continuation. The correlators taken to the boundary are natural diffeomorphism-invariant observables. Working at finite but large $r$ introduces some scheme dependence that presumably becomes controllable in the large $r$ limit.

\section{Can inflation begin by tunneling?}

After Farhi and Guth [26] established that beginning inflation classically required a past singularity, Farhi, Guth and Guven [27] (FGG) made the interesting proposal that 
inflation could be initiated by quantum tunneling. They computed a nonzero rate for this process using a Euclidean instanton. This rate was also derived using Hamiltonian techniques 64, 65] (see also [66]).

Roughly speaking, FGG envisioned a process where an initial state (a "buildable" state) was constructed by classical field evolution. This state would then undergo tunneling. The initial configuration would look like the bound trajectory on the left hand side of the effective potential29 (Fig. 2a). It would tunnel "through the effective potential" to the unbound inflating trajectory with the same energy on the right hand side.

A number of authors have argued that this process is not physically allowed. In particular, Banks [37] argued that since the de Sitter entropy of the inflating region is characteristically greater than the entropy of the black hole surrounding it, ideas of black hole complementarity and holography prohibit the process. Susskind [38 has given a somewhat different entropic argument that conflicts with the instanton rate.

The picture developed in this paper allows us to give a sharp argument against FGG tunneling, at least in the AdS context. The initial buildable state is clearly obtainable by unitary quantum time evolution, and so is a pure state. The final state has an inflating region, and so by the arguments in Section 3 is a mixed state. But unitary quantum evolution cannot take a pure state to a mixed state. So this process cannot occur. In fact, no state corresponding to inflation can ever result from any pure state process. This argument is close in spirit to that of [37], since the large entropy of the de Sitter region requires that the state be mixed. But the argument presented here is more general, since there exist time asymmetric situations where the de Sitter entropy is less than the black hole entropy where the state is mixed and hence creation by any process is ruled out.

Such a simple argument demonstrates the power of embedding a physical phenomenon in a well defined non-perturbative formalism. But it is still important to understand the loophole in the FGG argument.

\section{Discussion}

We started our discussion by assuming the existence of the string landscape, with many de Sitter and AdS vacua. We restricted our attention to a stable (supersymmetric) AdS vacuum and a neighboring de Sitter minimum. Focusing on the low energy gravity dynamics (and choosing points on the landscape, and hence parameters, appropriately) we solved for the geometry of the system using the thin wall approximation. As expected from previous work [26,27], we found parameter domains with inflating behavior behind a

29 In order to avoid singularity theorem constraints and hence be buildable the initial geometry must be (a small deformation) of the lower half of Fig. đ]b. 
black hole horizon. The stable AdS minimum should, by general arguments, be described by a boundary CFT. Because the inflating region involves an excitation of fields that exist in this minimum, it is plausible that it will also be described as an excitation of the CFT. One of our basic conclusions is that these inflating regions must be described by a mixed state, i.e. a density matrix in the CFT. Our strongest argument interpreted the static domain wall as a cutoff version of the eternal Schwarzschild-AdS black hole, a system known to be described by a mixed state. The inflating geometries will certainly be mixed if this one is. This mixed state description resolves an entropy puzzle because the large number of inflating degrees of freedom need not be explicitly represented in the CFT. This description raises several important questions. First, as we smoothly increase the size of the initial bubble, moving through the progression of geometries illustrated in Fig. 7, when does a mixed state become necessary for a CFT description? We have discussed several possible answers to this question but it still remains open. More generally, given a rather arbitrary mixed state in the CFT, what is its geometric interpretation? Finally, a striking aspect of this description is the necessity of using a mixed state to describe a geometry with one asymptotic region (as in the static domain wall example). Most previous examples requiring mixed state descriptions had other asymptotic (AdS) boundaries. They could be given a pure state description if all boundary degrees of freedom were kept. Mixed states resulted when some boundaries were traced over. Here we do not have an explicit representation of these extra degrees of freedom, although we know many of their properties. This provides a rather well controlled system in which to search for new, non-boundary, descriptions of non-perturbative quantum gravity.

We described techniques for probing the inflating region, even though the degrees of freedom there were not given explicitly. These techniques relied on analyticity. The first used geodesics. These geometries have nearly null geodesics that bounce off de Sitter $\mathcal{I}$, so they give subdominant singular contributions to certain correlators. To study them one must take $g_{s}$ very small, which suppresses bubble nucleation. Chaotic eternal inflation should still be visible with these probes. More generally one can continue correlators to complex time. We argued that AdS boundary correlators should continue to de Sitter boundary correlators in the classical limit. At finite $g_{s}$ the complex pattern of bubble nucleation and other non-perturbative processes should be visible in these continued correlators, which provide in principle a non-perturbative description of some aspects inflationary dynamics. Bubble nucleation is an $\exp \left(-1 / g_{s}^{2}\right)$ process and should represent the continuation of such a process. This should be an instanton in the CFT. Tunneling to an AdS vacuum will result in a big crunch, to a zero cosmological constant vacuum will result in an FRW cosmology. So this setup should give non-perturbative descriptions of these different cosmologies, albeit in an indirect way.

At first glance, it seems that to use these techniques to extract important informa- 
tion about inflation would require a rather explicit description of the boundary CFT. A boundary CFT capturing aspects of the landscape as a whole will be an extraordinarily complicated object. But perhaps it would be possible to define a truncated CFT that represents an effective bulk theory with a only a few minima. Higher order non-perturbative effects could possibly be systematically ignored.

Even without such a description we were able to draw some general conclusions, relying only on general features of the picture we have developed. In particular we were able to argue that inflating regions could not be produced, even by quantum mechanical tunneling, in a scattering process because a pure state cannot evolve into a mixed state under Hamiltonian time evolution. More results of this general type would certainly be welcome.

Acknowledgments: We would like to thank Tom Banks, Raphael Bousso, Petr Hořava, Gary Horowitz, Shamit Kachru, Matt Lippert, Juan Maldacena, Don Marolf, Simon Ross, Yasuhiro Sekino, Eva Silverstein, Lenny Susskind, and Erik Verlinde for stimulating discussions. VH and MR would also like to thank Gary Horowitz and Sandip Trivedi for initial collaboration on related issues. VH, AM, and MR would like to acknowledge KITP and the Fields Institute for hospitality during this work. VH and MR would also like to thank the organisers of the 2005 Amsterdam String theory workshop for hospitality. This work was supported in part by the Stanford Institute for Theoretical Physics, NSF grant PHY-9870115, the Department of Energy under contract DE-AC02-76SF00515, the funds from the Berkeley Center for Theoretical Physics, DoE grant DE-AC02-05CH11231, and the NSF grant PHY-0098840. In addition, research at the Perimeter Institute is supported in part by funds from NSERC of Canada and MEDT of Ontario. RCM is further supported by an NSERC Discovery grant.

\section{Appendix A. Details of the thin wall geometries}

In Section 2 we briefly outlined the construction of thin domain wall spacetimes and stated the main results for the specific case of interest, the de Sitter/Schwarzschild-AdS junction. In this appendix we will go into more detail and derive these results. As many of these results apply in a broader set-up than presented above, we first present the effective potential and extrinsic curvatures for a more general junction between two spacetimes, with two free parameters (the black hole mass and the cosmological constant) each.

\section{A.1. Effective potential and extrinsic curvatures}

We consider $D$-dimensional metrics of the form (2.1), (2.2), with

$$
f_{\alpha}(r)=1-\lambda_{\alpha} r^{2}-\frac{\mu_{\alpha}}{r^{D-3}}
$$


where $\alpha$ stands for $i$ or $o$. Here $\lambda$ is related to the cosmological constant, which can have either sign, and $\mu$ to the black hole mass. (Thus for example, $\lambda_{i}>0$ and $\lambda_{o}<0$ with generic $\mu_{\alpha}>0$ would correspond to the Schwarzschild-de Sitter/Schwarzschild-AdS junction.) The effective potential is

$$
\begin{aligned}
V_{\mathrm{eff}}(r)=-[ & \left.\lambda_{o}+\frac{\left(\lambda_{o}-\lambda_{i}-\kappa^{2}\right)^{2}}{4 \kappa^{2}}\right] r^{2}+1-\left[\mu_{o}+\frac{\left(\lambda_{o}-\lambda_{i}-\kappa^{2}\right)\left(\mu_{o}-\mu_{i}\right)}{2 \kappa^{2}}\right] \frac{1}{r^{D-3}} \\
& -\frac{\left(\mu_{o}-\mu_{i}\right)^{2}}{4 \kappa^{2}} \frac{1}{r^{2 D-4}}
\end{aligned}
$$

and the extrinsic curvatures are

$$
\begin{aligned}
& \beta_{i}(r)=\left(\frac{\lambda_{o}-\lambda_{i}+\kappa^{2}}{2 \kappa}\right) r+\left(\frac{\mu_{o}-\mu_{i}}{2 \kappa}\right) \frac{1}{r^{D-2}} \\
& \beta_{o}(r)=\left(\frac{\lambda_{o}-\lambda_{i}-\kappa^{2}}{2 \kappa}\right) r+\left(\frac{\mu_{o}-\mu_{i}}{2 \kappa}\right) \frac{1}{r^{D-2}},
\end{aligned}
$$

for this choice of geometries.

Let us consider some generic features of the effective potential (A.2). First, at small $r$, the last term dominates (provided $\mu_{o} \neq \mu_{i}$ as will be the case whenever the shell carries energy), implying $V_{\text {eff }}(r) \rightarrow-\infty$ as $r \rightarrow 0$. Hence it is always possible to have a shell which implodes to zero size. Such a shell crashes into the black hole singularity at $r=0$ in a finite proper time.

Secondly, at large $r$, the first term in (A.2) dominates (again, provided its coefficient does not vanish). The $r^{2}$ coefficient may have either sign, but noting that we can re-express it as

$$
-\left[\lambda_{o}+\frac{\left(\lambda_{o}-\lambda_{i}-\kappa^{2}\right)^{2}}{4 \kappa^{2}}\right]=-\frac{1}{4 \kappa^{2}}\left[\left(\lambda_{o}+\lambda_{i}+\kappa^{2}\right)^{2}-4 \lambda_{o} \lambda_{i}\right],
$$

we see that whenever the inside and outside cosmological constants have opposite sign, this coefficient is necessarily negative. This means that in such cases $V_{\text {eff }}(r) \rightarrow-\infty$ as $r \rightarrow \infty$, so that it is possible to have a shell which expands forever and (after infinite proper time) hits the boundary of both spacetimes. For $\lambda_{i}>0$ this describes inflation.

On the other hand, if the $r^{2}$ coefficient (A.4) of the effective potential is positive, then the boundary $r=\infty$ is not attainable by any shell, and a time symmetric situation is always possible. We can similarly read off the more detailed behavior of the shell by considering the extrinsic curvatures, as we do next in the 4-dimensional de Sitter/Schwarzschild-AdS context. This will justify the results presented in Section 2.

\section{A.2. Thin wall trajectories}

Focussing now on the specific case of 4-dimensional de Sitter/Schwarzschild-AdS junction given by (2.9), the effective potential (A.2) simplifies to (2.10). To see where the shell 
is allowed to appear on the appropriate Penrose diagrams, we will first consider the behavior in the vicinity of small and large $r$. As mentioned in Section 2, this is determined by the sign of the extrinsic curvatures on the two sides of the shell. These are given by (A.3), which for the de Sitter/Schwarzschild-AdS junction simplify to

$$
\beta_{i}(r)=\frac{\kappa^{2}-\lambda-1}{2 \kappa} r+\frac{\mu}{2 \kappa} \frac{1}{r^{2}}, \quad \beta_{o}(r)=-\frac{\kappa^{2}+\lambda+1}{2 \kappa} r+\frac{\mu}{2 \kappa} \frac{1}{r^{2}} .
$$

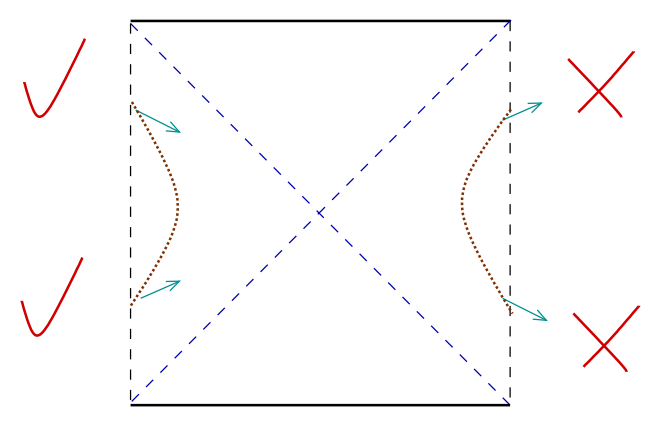

(a)

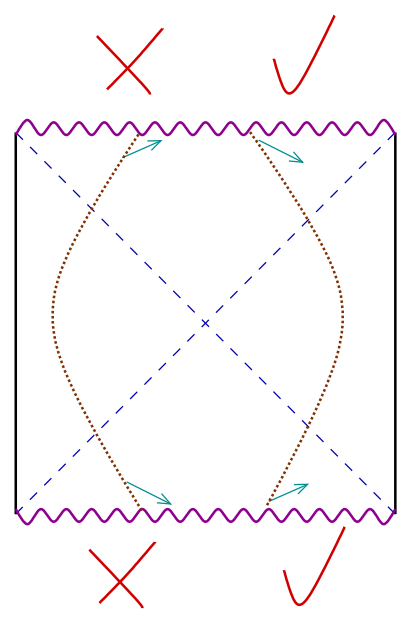

(b)

Fig. 10: Allowed and disallowed types of behavior of the de Sitter/SchwarzschildAdS domain wall at small $r$. The dotted curves depict the shell's trajectory (on (a) de Sitter and (b) Schwarzschild-AdS Penrose diagrams) and the arrows the correspond to the outward-pointing normal. The checks indicate allowed scenario while the crosses label disallowed scenario. (a) In de Sitter, the shell starting from or ending at the left origin is allowed, whereas starting/ending on the right origin is not allowed. (b) In Schwarzschild-AdS, the shell starting towards the right from the past singularity or moving towards the left before hitting the future singularity is allowed, whereas the opposite behavior is not allowed.

Clearly, as $r \rightarrow 0$, the second term dominates, and is always positive. Hence both $\beta_{i}(r) \rightarrow+\infty$ and $\beta_{o}(r) \rightarrow+\infty$ as $r \rightarrow 0$. This means that when the shell is sufficiently small, the outward-pointed normal has to point toward larger $r$. Fig. 10 summarizes the allowed and disallowed scenarios 30 On the de Sitter side (where the 'origin' $r=0$ are the

30 Although a complete shell trajectory is sketched in Fig. 10, only the $r \rightarrow 0$ part is of relevance - i.e., the full trajectory may or may not be allowed, based on additional constraints to be discussed later. Also, Fig. 10 is not intended to indicate the time of impact $t_{\alpha}(r \rightarrow 0)$ of the shell, but only the sign of $\frac{d t_{\alpha}}{d r}(r \rightarrow 0)$. 
north and south poles described by the vertical dashed lines in Fig. 10a), the normals point toward increasing $r$ only on the left side of the Penrose diagram. Hence the shell is allowed to hit only the left origin but not the right origin, as indicated in Fig. 10a. Similarly, for the Schwarzschild-AdS part of the spacetime, the shell can start out from the past singularity towards the right on the Penrose diagram, and hit the future singularity moving towards the left, but not vice-versa, as indicated in Fig. 10b.

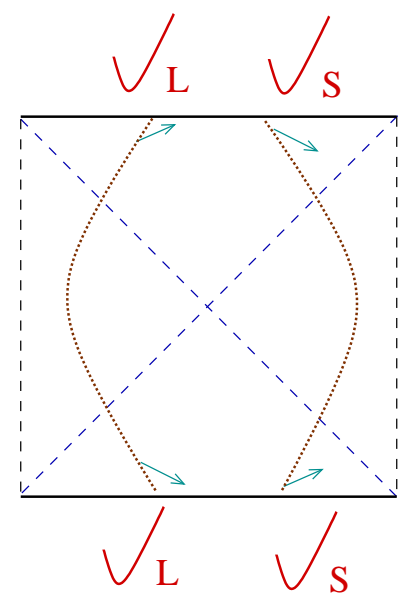

(a)

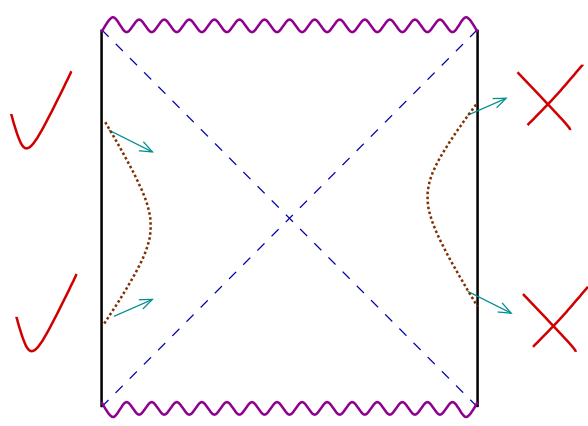

(b)

Fig. 11: Allowed and disallowed types of behavior of the de Sitter/SchwarzschildAdS domain wall at large $r$. (a) In de Sitter, the shell starting from de Sitter $\mathcal{I}^{-}$ towards the right or ending at $\mathcal{I}^{+}$veering left is allowed for small tension (namely $\left.\kappa^{2}<\lambda+1\right)$; whereas the opposite behavior is allowed for large tension $\left(\kappa^{2}>\lambda+1\right)$. (b) In Schwarzschild-AdS, the shell starting from or ending at the left boundary is allowed, whereas the the shell starting from or ending at the right boundary is not allowed.

At large $r$, the first terms in (A.5) dominate; here the sign of $\beta_{i}$ depends on the shell's tension and the de Sitter cosmological constant. For $\kappa^{2}<\lambda+1$, the first term is negative, so that $\beta_{i}(r) \rightarrow-\infty$ as $r \rightarrow \infty$, i.e., the outward normal from the de Sitter side points towards decreasing $r$. Conversely, for large tension $\kappa^{2}>\lambda+1$, the extrinsic curvature remains positive, so the outward normal points toward larger $r$. This is indicated in Fig. 11a, by the right and left trajectories, respectively. The corresponding behavior on Schwarzschild-AdS side, sketched in Fig. 11] b, is more universal: Here $\beta_{o} \rightarrow-\infty$ as $r \rightarrow \infty$ for all $\kappa$ and $\lambda>0$, so that the shell can hit the left boundary but not the right boundary.

We can now determine the full trajectory of the shell on the Penrose diagram. This requires us to solve for $R(\tau)$ using the expression for $V_{\text {eff }}$, while keeping in mind the sign of the extrinsic curvatures $\beta_{\alpha}$. These extrinsic curvatures are plotted along with the effective potential, for the various cases (A - E of Fig. 2) in Fig. 12. The parameters $\lambda$, $\mu$, and $\kappa$ 

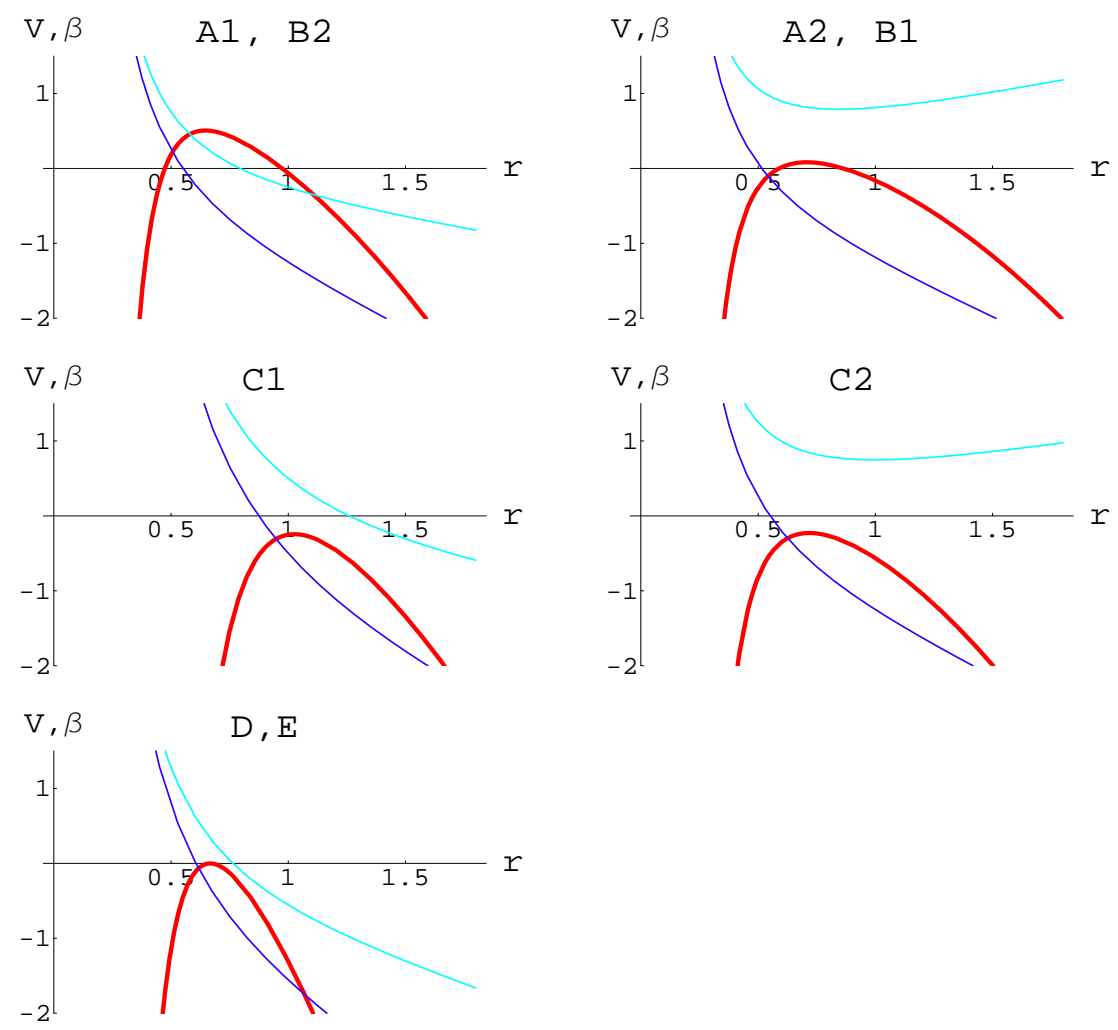

Fig. 12: Various possible effective potentials (thick, concave down curve) and extrinsic curvatures (thin, concave up curves, where $\beta_{i}(r)>\beta_{o}(r)$ ) describing the de Sitter/Schwarzschild-AdS junction. The specific parameters $(\lambda, \mu, \kappa)$ used were: $(\mathbf{A} 1, \mathbf{B 2}) \lambda=0.5, \mu=0.75, \kappa=2 ; \mathbf{( A 2 , B 1 )} \lambda=1, \mu=0.5, \kappa=1 ;(\mathbf{C 1})$ $\lambda=1, \mu=2, \kappa=1$; (C2) $\lambda=1, \mu=1, \kappa=2$; and (D,E) $\lambda=2, \mu=0.89, \kappa=1$.

are of order unity in all cases 31. The top two plots (labeled as cases A and B, consistently with the notation employed in Fig. 2) exemplify the situations with $V_{\max }>0$. For each potential there are two possible trajectories: (A) the shell expands from zero size and re-contracts and $(\mathrm{B})$ the shell contracts from infinite size and re-expands. The middle two plots in Fig. 12 (cases $\mathrm{C}$ ) describe a time asymmetric situation since $V_{\max }<0$. The two columns are distinguished by the sign of $\beta_{i}(r \rightarrow \infty)$. Finally, the bottom plot depicts a fine-tuned situation with $V_{\max }=0$.

Let us now consider the corresponding extrinsic curvatures, which will enable us to construct the full Penrose diagrams. As we can see from Fig. 12(A1), $\beta_{o}$ remains positive everywhere along the A trajectory, so that the shell's turning point must lie in region I

31 The parameters chosen to plot the effective potential and extrinsic curvatures in Fig. 12 are chosen to clarify the geometrical aspects and are not necessarily in the physically interesting regime. 
(right side) of the Schwarzschild-AdS Penrose diagram. Conversely, in Fig. 12(A2), $\beta_{o}$ becomes negative before $V_{\text {eff }}$ becomes positive, which implies that the shell must pass through region III rather than region I on the Schwarzschild-AdS Penrose diagram 32. Similarly, the sign of $\beta_{i}$ along the B trajectory distinguishes the cases (B1) and (B2). In the former case $\beta_{i}$ is positive, whereas in the latter it is negative. Finally, as a consistency check, one can verify that we cannot have $\beta_{i}$ becoming negative at smaller $r$ then where $V_{\text {eff }}$ becomes positive, or $\beta_{o}$ becoming negative at larger $r$ then where $V_{\text {eff }}$ becomes negative again 33 .

A few remarks are in order. First, where exactly the shell passes with respect to the bifurcation point of the horizons depends on the details of the set-up, which distinguishes the cases A1 from A2, or B1 from B2, but which are not drawn separately in Fig. 6; i.e., (A) of Fig. 6 would strictly speaking correspond only to (A1) of Fig. 4, etc.. Second, note that the Penrose diagrams corresponding to the time reverse cases C' and E' (in the notation of Fig. 2) would be obtained by vertically flipping the diagrams (C) and (E), respectively.

\section{Appendix B. False vacuum bubbles in scalar-gravity systems}

First, we will briefly review the general formalism for obtaining a full spacetime by using the initial-value $(3+1)$ formulation of GR. Given an initial slice, the induced 3metric, the corresponding extrinsic curvature, and the initial values and velocities of the matter fields, Einstein's equations split into two equations describing the evolution and imposing constraints on the initial data. We are interested in gravity coupled to a scalar field $\phi$ with a given potential $V(\phi)$. The action for the system will be taken to be:

$$
S=\int \sqrt{-g}\left[\frac{1}{2} R-\frac{1}{2}(\nabla \phi)^{2}-V(\phi)\right] .
$$

32 Note that for (A2), since the trajectory on the Schwarzschild-AdS diagram must still bend towards the left while passing through the left region, we would expect that this case can occur for much smaller region in the parameter space than the more typical case (A1). Indeed, we confirm that this is the case by plotting the potentials as in Fig. 12: we find that it is much harder to obtain $V_{\max }>0$ with the $\beta_{o}$ intercept occurring at smaller $r$ than the $V_{\text {eff }}$ intercept.

33 In particular, denoting the zero-intercepts of the extrinsic curvatures by $r_{\beta_{i}}$ and $r_{\beta_{o}}$, we find that $V_{\text {eff }}^{\prime}\left(r_{\beta_{i}}\right)=-2 \lambda\left(\frac{\mu}{1+\lambda-\kappa^{2}}\right)^{1 / 3}<0$, while $V_{\text {eff }}^{\prime}\left(r_{\beta_{o}}\right)=\left(3+\lambda+\kappa^{2}\right)\left(\frac{\mu}{1+\lambda+\kappa^{2}}\right)^{1 / 3}>0$. This automatically implies that if $V_{\max }>0$, then $V_{\text {eff }}\left(r_{\beta_{i}}\right)>0$ and $V_{\text {eff }}\left(r_{\beta_{o}}\right)<0$. Finally, by monotonicity of the extrinsic curvatures, this implies that the $\beta_{i}$ intercept occurs at larger $r$ than where $V_{\text {eff }}$ first becomes positive, and similarly that the $\beta_{o}$ intercept occurs at smaller $r$ than where $V_{\text {eff }}$ becomes negative again. 
Consider for concreteness a 4-dimensional, spherically symmetric spacetime, with a 3-dimensional spacelike initial slice $\Sigma_{0}$. Further assume the metric on $\Sigma_{0}$

$$
d s^{2}=\frac{d r^{2}}{1-\frac{2 m(r)}{r}}+r^{2}\left(d \theta^{2}+\sin ^{2} \theta d \phi^{2}\right)
$$

The unit normal to the surface $\Sigma_{0}$ will be taken to be $\left(\frac{\partial}{\partial t}\right)^{a}$. To describe how this hypersurface fits into the full spacetime, we have to prescribe the extrinsic curvature. For simplicity, we will choose the extrinsic curvature to be proportional to the induced metric on the Cauchy slice,

$$
K_{a b}=h(r) g_{a b}
$$

Furthermore, we have a scalar field $\phi$ with a potential $V(\phi)$. We denote the radial variation of $\phi$ by $\phi^{\prime}$, and the time evolution by $\dot{\phi}$. Specification of initial data will involve picking appropriate functions for $\phi(r), \dot{\phi}(r)$ and $h(r)$. The constraint equations are

$$
\dot{\phi} \phi^{\prime}=-2 h^{\prime}
$$

and

$$
2 m^{\prime}+m r \phi^{\prime 2}=\frac{r^{2}}{2}\left(\dot{\phi}^{2}+\phi^{\prime 2}+2 V-6 h^{2}\right) .
$$

Substituting (B.4) into (B.5) we find

$$
2 m(r)=\int_{0}^{r} \hat{r}^{2}\left(V-3 h^{2}+\frac{2 h^{\prime 2}}{\phi^{\prime 2}}+\frac{1}{2} \phi^{\prime 2}\right) e^{-\frac{1}{2} \int_{\hat{r}}^{r} \bar{r} \phi^{\prime 2} d \bar{r}} d \hat{r}
$$

Thus specifying the initial field profile $\phi(r)$ in a given potential $V(\phi)$ determines the mass function $m(r)$ on $\Sigma_{0}$ and thus the metric. Note that we can then find the total ADM mass in the usual way, by considering $m$ at large $r$ and possibly adding an appropriate counter-term.

We will now motivate the procedure for picking the correct initial data in a scalargravity system, which is guaranteed to have the desired features, de Sitter $\mathcal{I}$ and AdS boundary.

We first make a trivial remark about causality. If we take any spacetime $\left(\mathcal{M}, g_{a b}\right)$ and consider some spacelike a-chronal (and possibly compact) surface $\Sigma$ and impose the corresponding induced metric $h_{a b}$ and extrinsic curvature $K_{a b}$ on $\Sigma$ as our initial conditions, then within the domain of dependence of $\Sigma$, we are guaranteed to evolve to the spacetime $g_{a b}$. 


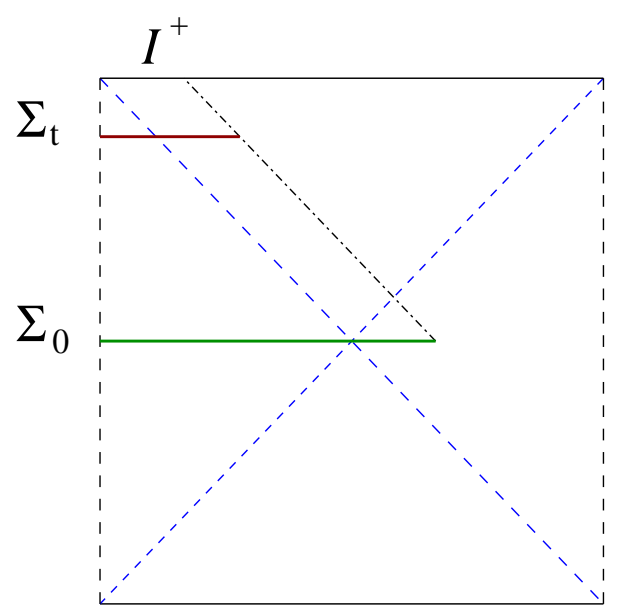

Fig. 13: Initial slices on a de Sitter Penrose diagram which are guaranteed to contain a piece of de Sitter $\mathcal{I}$ in their evolution. (The vertical lines are the poles $r=0$ of de Sitter, the dashed diagonal lines are the cosmological horizons $r=r_{d}$. Horizontal slices represent 3 -spheres, with their equator along the vertical midpoint of the diagram.)

This implies that if we want to consider (a piece of) initial data which is guaranteed to evolve to a de Sitter $\mathcal{I}^{+}$, we must take compatible initial conditions on a large enough initial surface (which spans the entire past domain of influence of the desired piece of de Sitter $\mathcal{I}$ ). This is illustrated on the de Sitter Penrose diagram in Fig. 13. If we wish to take the initial surface in a time-symmetric fashion (i.e. $\Sigma_{0}$ at $t=0$ ), then we must take $\Sigma_{0}$ to cover more than half of the sphere, in which case the radial coordinate is not monotonically increasing along the entire slice. On the other hand, if we consider a slice at some later time $t$, say $\Sigma_{t}$, we can easily contain the same piece of $\mathcal{I}^{+}$under evolution, while $\Sigma_{t}$ covers only a small part of the sphere and therefore has increasing radial coordinate. This makes the formalism sketched above applicable; in particular, we can use (B.6) to find the mass. Moreover, in a domain wall construction, if we imagine setting up a (non-static) domain wall to pass at the right end of $\Sigma_{t}$ in Fig. 13, we no longer have the requirement that the de Sitter size has to be bigger than that of the black hole: all we need to satisfy is that $R(t)>r_{d}$ and $R(t)>r_{+}$at the time $t$. Such constructions are discussed in detail in Appendix C.

Let us now see more explicitly how to construct a spacetime containing both de Sitter and AdS using a scalar field in a potential. We use the potential $V(\phi)$ as sketched in Fig. 14a. Note that if the field $\phi(r)$ were homogeneous and sat in the positive local minimum of the potential, namely $\phi(r)=0$, the spacetime would correspond to a static, spherically symmetric spacetime with a positive cosmological constant, namely de Sitter. Likewise, if the field sat in the negative local minimum of the potential, $\phi(r)=\phi_{1}$, the spacetime would correspond to static, spherically symmetric spacetime with a negative 


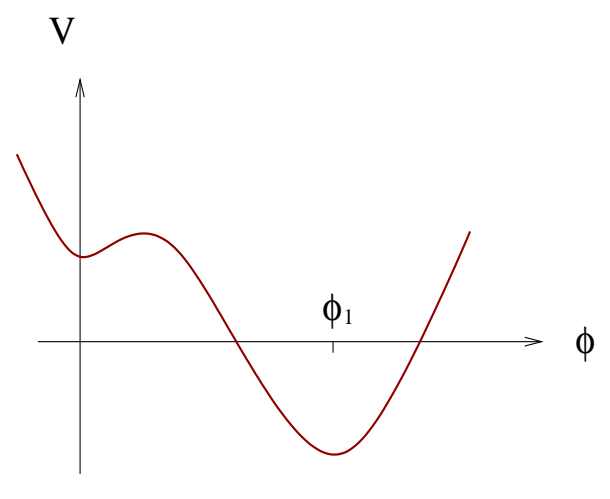

(a)

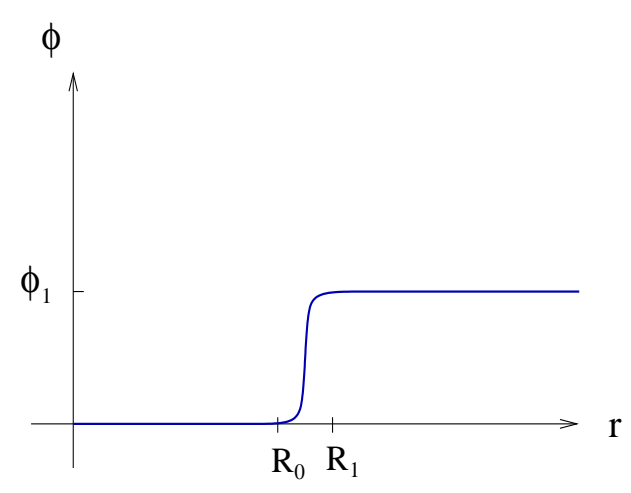

(b)

Fig. 14: (a) The potential $V(\phi)$ and (b) an initial profile of the field $\phi(r)$.

cosmological constant, namely AdS or Schwarzschild-AdS, depending on the mass.

To interpolate between de Sitter and Schwarzschild-AdS, consider the field profile as sketched in Fig. 14 $\mathrm{b}$, with $\dot{\phi}=0$ in the regions $r<R_{0}$ and $r>R_{1}$. For $r<R_{0}$, the field sits in the positive local minimum of the potential, so that in the domain of dependence of $r<R_{0}$, the spacetime corresponds to a part of the de Sitter spacetime. By arranging the corresponding initial surface $\Sigma_{t}$ to be late enough, this will include a part of the de Sitter $\mathcal{I}^{+}$, as in Fig. 13. Similarly, for $r>R_{1}$, the field sits in 34 the negative local minimum of the potential, corresponding to a part of Schwarzschild-AdS spacetime.

On a spacetime diagram, the full evolution might look like the one sketched in Fig. 15. Inside the domain of dependence of $r<R_{0}$ (left wedge), we obtain de Sitter $\mathcal{I}^{+}$. Similarly, inside the domain of dependence of $r>R_{1}$ (right wedge), we have Schwarzschild-AdS spacetime. Depending on the mass (in part determined by the size of the de Sitter region), the evolution may or may not reach a singularity. We note in passing that if there is a singularity in this region, as assumed in Fig. 15, then it will be of the Schwarzschild-AdS type, i.e., spacelike geodesics will bounce off the singularity. We also note that in order to have $r$ increasing monotonically on $\Sigma_{t}$, we cannot have $\Sigma_{t}$ go through region 3 of SchwAdS; this implies that in Fig. 15 there can be future or past Schwarzschild-AdS singularity,

34 There is a slight subtlety: if we require the profile $\phi(r)$ to be an analytic function of $r$, there has to be a slight deviation from $\phi$ being exactly constant in the regions $r<R_{0}$ and $r>R_{1}$. For de Sitter, this deviation does not change our arguments, since de Sitter is stable. On the other hand, the AdS minimum is more sensitive: if a homogeneous field is slightly off the minimum, it will big crunch. However, here we don't have homogeneous fields, and we can tune the deviation from the minimum to be infinitesimal, which implies by the well-posedness of the initial value formulation in general relativity that the resultant spacetime should be arbitrarily close to Schwarzschild-AdS. 


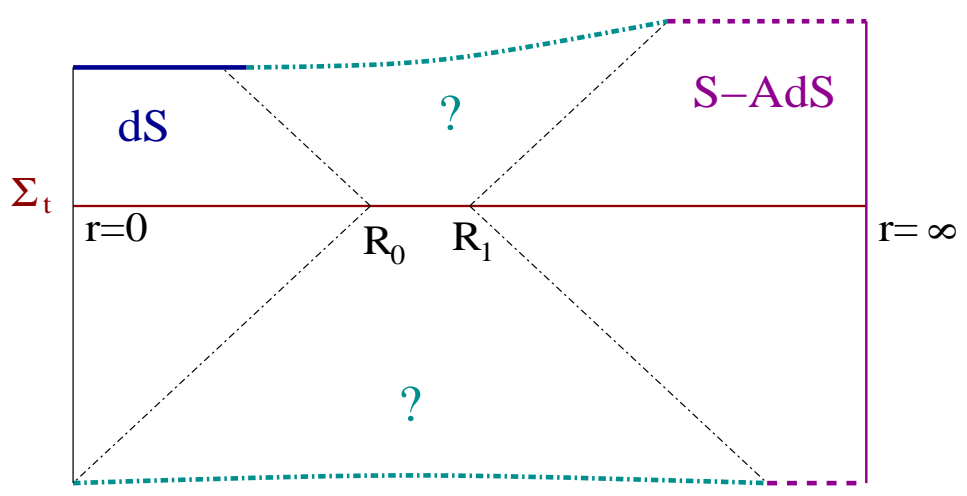

Fig. 15: Sketch of possible causal diagram for spacetime evolved from the initial data of Fig. 14.

but not both. The regions marked by '?' in Fig. 15 refers to the domain of dependence of the interpolating region $r \in\left[R_{0}, R_{1}\right]$ in the scalar profile Fig. 14] $\mathrm{b}$, for which the evolution needs to be determined numerically.

The above arguments illustrate that, at least within the realm of classical general relativity coupled to a scalar field, we can obtain de Sitter $\mathcal{I}$ within an asymptotically AdS spacetime.

\section{Appendix C. Construction allowing de Sitter $\mathcal{I}$ and $r_{d}<r_{+}$}

In this Appendix, we build on the set-up introduced in Appendix B to give a rigorous construction of spacetimes with de Sitter entropy smaller than the black hole entropy. We have indicated in Section 2 that for time asymmetric configurations it is possible to obtain $r_{d}$ both larger and smaller than $r_{+}$, depending on the parameters. In this Appendix we will demonstrate that in fact, geometries with $r_{d}<r_{+}$are not only possible, but indeed required, if the initial slice $\Sigma$ has monotonically increasing $r$ and the de Sitter $\mathcal{I}$ is guaranteed by evolution from $\Sigma$.

Consider the thin domain wall spacetimes separating de Sitter and SchwarzschildAdS, compatible with an initial surface $\Sigma$ on which $r$ increases monotonically. Recall from (A.5) that both the de Sitter and the Schwarzschild-AdS extrinsic curvatures are positive for small $r$ and negative for large $r$ (though $\beta_{i}(r \rightarrow \infty)$ depends on $\kappa$; but this will not affect our discussion). Moreover, since $\beta_{i}(r)>\beta_{o}(r)$ for all $r$ (which follows from that requirement that the bubble have positive energy), the respective radii $r_{\beta_{i}}$ and $r_{\beta_{o}}$ where the de Sitter and Schwarzschild-AdS extrinsic curvatures vanish must be related as $r_{\beta_{i}}>r_{\beta_{o}}$. We will now spell out the sequence of steps to determine the Penrose diagram: - To have an initial slice $\Sigma$ with monotonic $r$, the shell cannot be confined entirely to the right wedge of de Sitter. Likewise, it cannot be confined entirely to the left wedge of Schwarzschild-AdS. More specifically, $\Sigma$ cannot enter into these regions. This is because 
we want $r$ to increase towards the right on the initial slice, whereas it would necessarily increase towards the left in the above regions.

- To ensure de Sitter $\mathcal{I}^{+}$by causality arguments, the shell must end up at $r=\infty$. Evidently, the shell's trajectory must intersect the boundary of the past domain of influence of the desired piece of $\mathcal{I}^{+}$; this implies that it must pass through the upper and/or right wedge of de Sitter. But since $\Sigma$ cannot enter into the right wedge of de Sitter, the shell must pass through the upper wedge. This ensures that the shell must end up at $\mathcal{I}^{+}$since it follows a timelike trajectory.

- In order for the shell to end up at $r=\infty$, it must end up in the left wedge of Schwarzschild-AdS (and in the upper wedge of de Sitter, as explained in the previous point). Since $r$ is continuous across the shell, the shell must end on one of the AdS boundaries. The negativity of the AdS extrinsic curvature at large $r$ implies that the shell cannot end up at the right boundary, since this would require the extrinsic curvature to be positive (the outward normal would necessarily have to point to larger $r$ ).

- In order for the shell to end up in the left wedge of Schwarzschild-AdS, it cannot pass through the right wedge of Schwarzschild-AdS. This is implied simply by the fact that the shell follows a timelike trajectory.

- The first and the last points imply that the shell must pass through the lower wedge of Schwarzschild-AdS. In fact, since the shell follows a timelike trajectory, this further implies that it must start in the lower wedge of Schwarzschild-AdS, i.e., at the singularity, $r=0$. Note that this requires that the effective potential for the domain wall motion be negative semi-definite, $V_{\text {eff }}(r)<0$ for all $r$, which is easy to achieve for suitable choices of parameters.

- Finally, in order for the shell to start at $r=0$ and therefore the de Sitter extrinsic curvature be positive, it must start in the left wedge of de Sitter. This is again implied by the incompatibility of extrinsic curvature with starting at the origin in the right wedge of de Sitter: for any such scenario, the de Sitter extrinsic curvature would have to be negative, since the outward normal would point toward decreasing $r$.

Thus far, we have established that from the de Sitter point of view, the shell must start on the left origin and end on the upper $\mathcal{I}^{+}$; whereas from the Schwarzschild-AdS point of view, it must start at the past singularity and end up on the left boundary. To refine this picture, we can use the facts about the extrinsic curvatures further to say that the shell must start out going to the right and end up going to the left on the Penrose diagram. The complete trajectory is illustrated in Fig. 16.

Finally, if we combine these on a single Penrose diagram and draw in the initial surface $\Sigma$, the resulting diagram would look like Fig. 17. Note that the de Sitter horizon crosses the shell's trajectory lower (i.e., at smaller $r$ ) than the black hole horizon, which implies that $r_{d}<r_{+}$. Also note that on Fig. 16, the initial slice $\Sigma$ would cut the de Sitter Penrose 


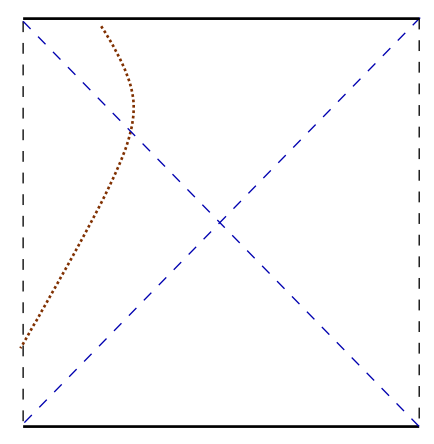

$\mathrm{dS}$

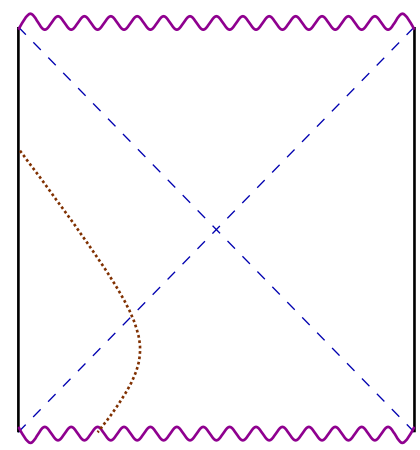

Schw-AdS

Fig. 16: The domain wall trajectory on de Sitter and Schwarzschild-AdS Penrose diagrams which is necessitated by the type of set-up specified above: and initial slice $\Sigma$ with monotonically increasing $r$, and a guarantee of dS $\mathcal{I}^{+}$under evolution.

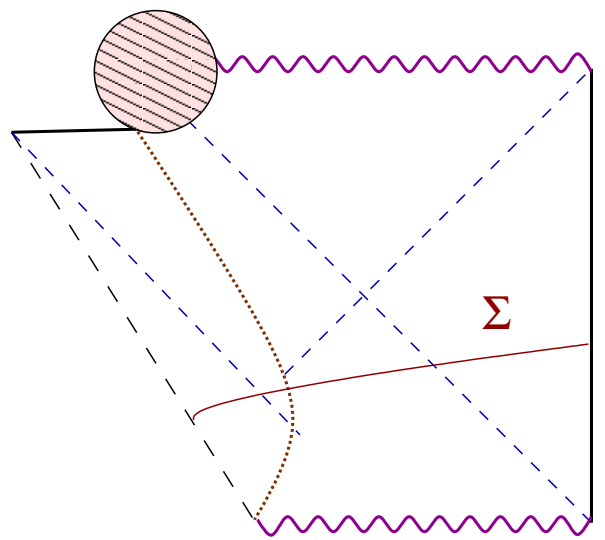

Fig. 17: Initial slice $\Sigma$ with monotonically increasing $r$ on a combined Penrose diagram (obtained by taking the left part of dS and right part of S-AdS in Fig. 16). The shaded region is uncertain under a reasonable evolution.

diagram in the upper wedge whereas it would cut the Schwarzschild-AdS Penrose diagram in the lower wedge. While $\Sigma$ in Fig. 17 has $r$ monotonically increasing, we this property does not continue to hold for all later spacelike slices which would cover the full Penrose diagram. In this sense, Fig. 15 is somewhat misleading: $r$ is not a good coordinate globally.

¿From the above arguments we see that Fig. 16 represents the only self-consistent setup with thin domain wall, and initial slice with monotonically increasing $r$ and a guaranteed piece of dS scri under evolution. Explicitly, in order for $r$ to increase monotonically on our initial data slice $\Sigma$, we must have $R_{1}<r_{+}$. On the other hand, to guarantee that the domain of dependence of the part of $\Sigma$ with $r<R_{0}$ includes a part of dS scri, we must have $R_{0}>r_{d}$. Since according to our setup, $R_{0}<R_{1}$, the two conditions together guarantee that the de Sitter radius (and therefore its entropy) is smaller than that of the 
black hole.

Although the above argument has been phrased in the thin wall context, it actually applies in general: assuming $r$ is monotonic on the Cauchy slice, in order to guarantee de Sitter $\mathcal{I}^{+}$within the domain of dependence of our Cauchy slice, we require $r_{d}<r_{+}$.

\section{Appendix D. A pure state description of spacetimes with causally disconnected regions?}

In section 4 , one of the criteria we proposed for the boundary field theory to be in a mixed state was that the spacetime geometry have regions that are causally disconnected from the boundary. However, in some special cases we can construct spacetimes containing a causally inaccessible region which nevertheless appear to have a pure state description in the CFT. In this section we will describe two examples of this. In both cases, a subset of CFT correlators are insensitive to the causally disconnected region and act as though they are in a mixed state.

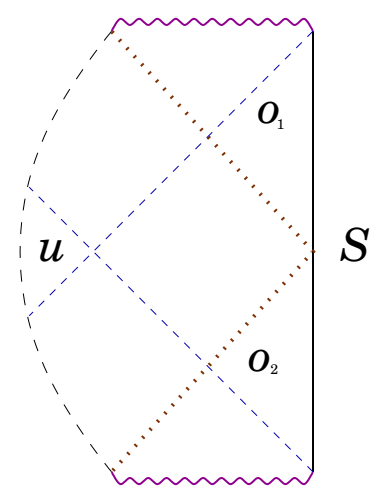

Fig. 18: Sketch of possible causal diagram for collapse spacetime created by an imploding null shell in AdS. Here the spacetime inside the shell is pure AdS.

The simplest example is a spherically symmetric null shell of energy which is launched from the boundary at an instant of time, say $t=0$. For simplicity we construct a timesymmetric geometry, so the shell is launched towards the future and the past, as shown in Fig. 18. The overall picture, then, is of a null shell expanding out to the boundary, reflecting off, and collapsing back in. Since the usual AdS boundary conditions are reflecting, such a configuration can be constructed as a solution of the source-free equations of motion.

The overall structure of the spacetime depends on the mass of the shell. If the collapsing shell is massive enough to make a stable black hole in AdS (one with $r_{+}>r_{A}$ so as to be stable in the canonical ensemble), then the spacetime has a region which is causally disconnected from the boundary, as shown in Fig. 18. By making the mass of the shell large, we can make the inaccessible region large in AdS units. 
To prove the existence of an inaccessible region, note that inside the null shell the metric is pure AdS, while outside it is Schwarzschild-AdS. If light rays emitted radially from the origin at $t=0$ escape out to the boundary, every other point in the spacetime will be accessible to the boundary. By "accessible," we mean that every bulk point is either in the future lightcone or the past lightcone of at least one boundary point. On the other hand, if radial light rays launched from $t=0, r=0$ end in the singularity, there exists a spacetime region which is causally disconnected from the boundary. The light ray of interest propagates in pure AdS inside the shell, so we only need to know the behavior of null geodesics in AdS to infer causal accessibility.

The question therefore is whether the radius $r_{0}$ at which the light ray crosses the shell is bigger or smaller than the Schwarzschild radius of the black hole. If it is smaller, then the light ray must match onto a point inside the horizon and end in the singularity. It turns out that the radius $r_{0}$ at which the light ray intersects the shell is precisely the AdS radius $r_{A}$. So anytime we form a stable black hole with $r_{+}>r_{A}$ the light rays from the origin will be unable to escape to the boundary and we have an inaccessible region. To prove that $r_{0}=r_{A}$, it suffices to note that in pure AdS spacetime two radial null geodesics, one originating at the origin $r=0$ and the other at the boundary meet precisely at $r=r_{A}$. It is easy to check that for pure AdS, $r_{\text {ray }}(t)=r_{A} \tan \left(t / r_{A}\right)$ and $r_{\text {shell }}(t)=r_{A} \cot \left(t / r_{A}\right)$, respectively, which intersect at $r=r_{A}$. As an aside, it is possible to show that time symmetric collapse geometries with $r_{+}>r_{A}$ always contain a region causally disconnected from the AdS boundary.

There are strong reasons to believe that this solution can correspond to a pure state in the CFT. On the time symmetric slice, the shell solution differs from pure AdS only by the excitation of some massless fields near the boundary. We can think of creating the state by acting on the vacuum with some bulk operators near the boundary. Since bulk operators near the boundary are easy to map the the field theory, in the CFT we should also be able to obtain this state by acting with an operator on the vacuum, which manifestly gives a pure state. We can imagine exciting the massless fields in the bulk by the insertion of an operator $\mathcal{S}$ in the boundary at time $t=0$; the geometry in Fig. 18 corresponds to a state $\mathcal{S}|0\rangle$ of the field theory. For example, we can construct a null shell with the desired properties by a dilaton wave packet in the bulk, which in t he boundary theory correpsonds to the choice

$$
\mathcal{S}=\exp \left(i \int d^{3} \vec{x} d t j(t) \operatorname{Tr}\left(F_{\mu \nu}(x, t) F^{\mu \nu}(x, t)\right)\right) .
$$

By picking the support $j(t)$ we can ensure that we have a configuration with null shell that is launched symmetrically away from the boundary and localized in time near $t=0$.

Although the field theory is in a pure state, the most obvious field theory operators are insensitive to physics in the inaccessible region, suggesting a mixed state. At the level of 
bulk field theory, the insertion of unitary operators $\mathcal{U}$ with support only in the inaccessible region will not affect spacelike separated operators. Consider the bulk correlation functions (see Fig. 18):

$$
\left\langle 0\left|\mathcal{S}^{\dagger} \mathcal{O}_{1} \mathcal{O}_{2} \cdots \mathcal{O}_{n} \mathcal{S}\right| 0\right\rangle \quad \text { and } \quad\left\langle 0\left|\mathcal{S}^{\dagger} \mathcal{U}^{\dagger} \mathcal{O}_{1} \mathcal{O}_{2} \cdots \mathcal{O}_{n} \mathcal{U} \mathcal{S}\right| 0\right\rangle
$$

If $\mathcal{U}$ is unitary and spacelike separated from all the $\mathcal{O}_{n}$, bulk locality guarantees that $\left[\mathcal{U}, \mathcal{O}_{n}\right]=0$; hence the two correlation functions in (D.2) are identical (using $\mathcal{U}^{\dagger} \mathcal{U}=$ 1). Taking the operators $\mathcal{O}_{n}$ out to the boundary we can recover boundary correlation functions using the usual AdS/CFT rules. So boundary correlators which are obtained as limits of bulk correlators are not sensitive to excitations in the causally inaccessible region. (Note that this argument is specific to insertion of unitary operators in the causally inaccessible region; we can not reach the same conclusion with Hermitian operators.) Thus correlation functions of local operators can be calculated by explicitly tracing over the inaccessible region, implying that they are evaluated in a mixed state in the boundary description.

Nonlocal operators, such as Wilson loops, appear to be able to detect the physics of the inaccessible region. In fact, since the $t=0$ slice is identical to pure AdS except for excitations at infinity, one expects that the holographic mapping is unchanged and so large Wilson loops should be able probe the "inaccessible" region 35 . The CFT would have to have the following strange property. In the state $\mathcal{S}|0\rangle$, the bulk excitations corresponding to $\mathcal{U}$ should presumably be captured by excitations of nonlocal operators. The strange feature of these excitations is that they cannot affect any correlation function of local gauge-invariant operators at any time. It is unclear whether the field theory needs to have this property exactly, since our argument relies on bulk locality and thus may only be approximately true. If we were to focus only on correlation functions of local operators in the CFT, we would see a mixed state which describes physics outside the horizon.

A second example of a spacetime with causally disconnected regions that appears to be described by a pure state involves the "Swedish Geons" of [67, 68, 69, 28,,70]. These spacetimes are orbifolds of $\mathrm{AdS}_{3}$, which have multiple boundaries and future and past singularities, much like the BTZ black hole. However, unlike the BTZ black hole, these geometries have regions in the interior that are causally disconnected from the all of the boundaries. In fact, the casual wedge associated to each boundary is metrically identical to the casual wedge of the BTZ black hole. There is a reasonably straightforward construction of a pure state in these spacetimes, following the Hartle-Hawking construction of [28]. These spacetimes have a simple Euclidean section, whose boundary is a higher genus

35 While the choice of time slicing is not unique, one expects to be able to map bulk operators to CFT operators on each time slice. 
Riemann surface. The Hartle-Hawking state, which is a pure state entangling degrees of freedom living on the various boundaries, is found by performing a path integral over this Riemann surface. The arguments above involving unitary operators will apply to these spacetimes as well.

\section{Appendix E. Analyticity in Coleman-de Luccia spacetimes}

As an example of continuing correlators from the AdS to de Sitter boundaries, it is useful to consider the relatively simple example of Coleman and de Luccia [62]. Although once backreaction is included the AdS boundaries are removed, if one ignores backreaction this simple geometry is an instructive toy model where many calculations can be done explicitly. We will focus on the three dimensional case, but most of our formulas are easily be extended to higher dimensional cases. We start with the Euclidean Coleman-De Luccia geometry

$$
d s^{2}=d \rho^{2}+f(\rho) d \Omega_{2}^{2},
$$

where $\rho$ runs from 0 to $\pi r_{d}$. The geometry approaches Euclidean AdS (the hyperboloid) as $\rho \rightarrow 0$, and Euclidean de Sitter (the sphere) $\rho \rightarrow \pi r_{d}$. So

$$
f(\rho)=\left\{\begin{array}{lc}
r_{a}^{2} \sinh ^{2} \frac{\rho}{r_{a}}, & \text { as } \rho \rightarrow 0 \\
r_{d}^{2} \sin ^{2} \frac{\rho}{r_{d}}, & \text { as } \rho \rightarrow \pi r_{d}
\end{array}\right.
$$

where $r_{a}$ and $r_{d}$ denote the AdS and de Sitter radii, respectively. The form of $f(\rho)$ depends on the profile of the domain wall. In the thin wall approximation, $f$ is found by matching the two asymptotic functions (E.2) at an intermediate value of $\rho$. In actuality, $f(\rho)$ is a complicated function obeying (E.2), whose exact form depends on the details of the domain wall.

If we Wick rotate one of the angles of the sphere, so that

$$
d \Omega_{2}^{2}=d t_{E}^{2}+\cos ^{2} t_{E} d \theta^{2} \rightarrow-d t^{2}+\cosh ^{2} t d \theta^{2},
$$

becomes the metric on a 2 dimensional de Sitter space, then (E.1) describes a Lorentzian geometry interpolating between AdS and de Sitter. Note that when we apply (E.3) to (E.1), we obtain AdS and de Sitter coordinate patches whose constant $\rho$ slices are copies of $d S_{2}$. These patches cover only a portion of the full geometry, as we will describe below.

We can now evaluate the correlators of a quantum field $\phi$ in this background. The geometry (E.1) is non-singular, so can be used to define a Euclidean vacuum state, in which correlators are defined by Wick rotation from Euclidean correlators on (E.1). In the 
limit where the mass of the scalar field is large, these Euclidean two point functions may be evaluated in position space as

$$
\left\langle\phi\left(x_{1}\right) \phi\left(x_{2}\right)\right\rangle \sim e^{-m \mathcal{L}\left(x_{1}, x_{2}\right)},
$$

where $\mathcal{L}\left(x_{1}, x_{2}\right)$ is the proper length of the geodesic between $x_{1}$ and $x_{2}$. In the AdS and de Sitter limits given by $(\mathbb{E} .2)$ this geodesic length is

$$
\mathcal{L}= \begin{cases}\cosh ^{-1}\left(r_{a}^{2}\left(\cosh \frac{\rho_{1}}{r_{a}} \cosh \frac{\rho_{2}}{r_{a}}-\sinh \frac{\rho_{1}}{r_{a}} \sinh \frac{\rho_{2}}{r_{a}} \cos \ell_{2}\right)\right), \quad \text { as } \rho_{1}, \rho_{2} \rightarrow 0 \\ \cos ^{-1}\left(r_{d}^{2}\left(\cos \frac{\rho_{1}}{r_{d}} \cos \frac{\rho_{2}}{r_{d}}+\sin \frac{\rho_{1}}{r_{d}} \sin \frac{\rho_{2}}{r_{d}} \cos \ell_{2}\right)\right), & \text { as } \rho_{1}, \rho_{2} \rightarrow \pi r_{d}\end{cases}
$$

where $\ell_{2}$ is the angular separation on the 2 sphere. In the interior,

$$
\mathcal{L}=\int_{\rho_{1}}^{\rho_{2}} \frac{d \rho}{\sqrt{1-L^{2} / f^{2}}},
$$

where $L$ is the conserved angular momentum of a geodesic,

$$
L^{2}=f^{4}\left(\dot{t}^{2}+\cos ^{2} t \dot{\theta}^{2}\right)
$$

which is related to the $\ell_{2}$ by

$$
\ell_{2}=\int \frac{d \rho}{f \sqrt{f^{2} / L^{2}-1}}
$$

The expression (E.6) is an analytic function as one moves from the AdS to the de Sitter regions, since the matching function $f(\rho)$ is analytic. So it allows us analytically continue correlators through the domain wall. In fact, for particular forms of $f(\rho)$, we can even continue correlators from the AdS boundary to the de Sitter $\mathcal{I}$.

This requires an extra step, since the Wick rotation (E.3) of the metric (E.1) gives us only certain patches of $\mathrm{AdS}_{3}$ and $\mathrm{dS}_{3}$, respectively, whose constant $\rho$ slices are copies of $\mathrm{dS}_{2}$. Thus to go from the patch of $\mathrm{AdS}_{3}$ containing the domain wall to another patch that contains the asymptotic AdS boundary we must analytically continue in both $t$ and $\rho$. To see this, recall that $\mathrm{AdS}_{3}$ may be written in terms of de Sitter slices as

$$
d s^{2}=d \rho^{2}+r_{a}^{2} \sinh ^{2} \frac{\rho}{r_{a}}\left(-d t^{2}+\cosh ^{2} t d \theta^{2}\right)
$$

The size of the de Sitter slices, $r_{a}^{2} \sinh ^{2} \rho / r_{a}$, goes to zero as $\rho \rightarrow 0$, indicating that the $\mathrm{dS}_{2}$ slices are becoming null. One may continue across this horizon to a patch foliated by hyperbolic slices

$$
d s^{2}=-d \rho^{\prime 2}+r_{a}^{2} \sin ^{2} \frac{\rho^{\prime}}{r_{a}}\left(d t^{\prime 2}+\sinh ^{2} t^{\prime} d \theta^{2}\right)
$$


by taking $\rho \rightarrow \rho^{\prime}=i \rho$ and $t \rightarrow t^{\prime}=t+i \pi / 2$; note that the shift in $t$ is necessary to keep $d \theta^{2}$ spacelike 36 . The $\mathrm{dS}_{2}$ slice that becomes null as $\rho \rightarrow 0$ matches onto an $H_{2}$ slice that becomes null as $\rho^{\prime} \rightarrow 0$. Then one can evolve forward in $\rho^{\prime}$ until the hyperbolic slice shrinks again to zero size as $\rho^{\prime} \rightarrow \pi r_{a}$. One then crosses this horizon by a similar analytic continuation to find a second coordinate system of the form (E.9). This second patch is related to the original coordinate patch by $t \rightarrow t^{\prime \prime}=t+i \pi$ and $\rho \rightarrow \rho^{\prime \prime}=\rho+i \pi r_{a}$. The $\left(\rho^{\prime \prime}, t^{\prime \prime}\right)$ patch contains an asymptotic AdS boundary at $\rho^{\prime \prime} \rightarrow \infty$.

Since constant $\rho^{\prime \prime}$ slices are copies of $\mathrm{dS}_{2}$, the AdS/CFT correspondence in these coordinates yields a dual boundary CFT living on $\mathrm{dS}_{2}$; this is in contrast to the more familiar examples of the sphere or the plane in global and Poincaré coordinates, respectively.

Of course, as mentioned above, when we go beyond the thin wall approximation (as is necessary to obtain analytic correlators) the geometry will typically develop a big crunch singularity before one can reach the AdS boundary. This is because the patch (E.10) has a surface of infinite blueshift at $\rho^{\prime} \rightarrow \pi r_{a}$, so any matter present will cause a strong backreaction. However, there certainly exist choices of analytic function $f(\rho)$ where this is not the case. In these cases the metric is completely smooth in all of the patches described above. Although such an $f(\rho)$ will not typically solve the appropriate equations of motion, this case still presents an interesting toy model where analytic continuation can be studied explicitly. We should emphasize that in the full de Sitter-Schwarzschild-AdS spacetimes the AdS boundary to the right of the black hole horizon is not removed by backreaction. So an analytic continuation similar to the one described here should apply.

To go from the $\rho \rightarrow \pi r_{d}$ region of the de Sitter patch of $\mathrm{dS}_{3}$ out to $\mathcal{I}^{+}$we must analytically continue in both $t$ and $\rho$. In particular, the patch of $\mathrm{dS}_{3}$ foliated by copies of $\mathrm{dS}_{2}$, with

$$
d s^{2}=d \rho^{2}+r_{d}^{2} \sin ^{2} \frac{\rho}{r_{d}}\left(-d t^{2}+\cosh ^{2} t d \theta^{2}\right)
$$

has a horizon at $\rho \rightarrow \pi r_{d}$. By taking $t \rightarrow t^{\prime \prime \prime}=t+i \pi / 2$ and $\rho \rightarrow \rho^{\prime \prime \prime}=i \rho-\pi R_{d}$ this horizon may be patched on to a region of $\mathrm{dS}_{3}$ with hyperbolic slices

$$
d s^{2}=-d \rho^{\prime \prime \prime 2}+r_{d}^{2} \sinh ^{2} \frac{\rho^{\prime \prime \prime}}{r_{d}}\left(d t^{\prime \prime \prime 2}+\sinh ^{2} t^{\prime \prime \prime} d \theta^{2}\right)
$$

The de Sitter boundary is found by taking $\rho^{\prime \prime \prime} \rightarrow \infty$. Note that in this coordinate system the boundary is a copy of $\mathrm{H}_{2}$; this $\mathrm{H}_{2}$ covers only part of the full de Sitter boundary $\mathbf{S}^{2}$ that one finds in global coordinates. Correlators of bulk fields in this coordinate system define boundary dS/CFT correlators on $\mathrm{H}_{2}$, as opposed to boundary correlators on the sphere or the plane described by [19]. Putting this together with (E.6), we have an explicit

36 In this section we will be careful to label the various $(\rho, t)$ coordinates in different patches of the spacetime by primes to emphasize that they describe different coordinate systems. 
analytic continuation of two point functions from points near an AdS boundary to points near a de Sitter boundary.

The considerations described above imply that boundary CFT correlators on the AdS boundary have very interesting analytic behavior. Expression (E.5) tells us that near the AdS boundary correlators are found by continuing $t \rightarrow t+\pi, \rho \rightarrow i \pi r_{a}$, and taking the large $\rho_{1}, \rho_{2}$ limit. Stripping off the factors of $e^{\rho_{1}}$ and $e^{\rho_{2}}$, this gives us the usual form for conformal two point functions on $\mathrm{dS}_{2}$,

$$
\left\langle\mathcal{O}_{\phi}\left(x_{1}\right) \mathcal{O}_{\phi\left(x_{2}\right)}\right\rangle \sim\left(\frac{1}{\sin ^{2} \ell_{2} / 2}\right)^{m}+\text { subleading },
$$

where $\ell_{2}$ is now the geodesic length on $\mathrm{dS}_{2}$. In the small $\ell$ limit this gives the usual short distance behavior $37 \ell_{2}^{-2 m}$. The more complicated form at finite distance is the standard formula for conformal two point functions on $\mathrm{dS}_{2}$; it can be thought of as arising from the Weyl anomaly for conformal field theories on de Sitter backgrounds. Near the de Sitter boundary, a similar prescription can be used to define correlators of a Euclidean conformal field theory on $\mathrm{H}_{2}$

$$
\left\langle\mathcal{O}_{\phi}\left(x_{1}\right) \mathcal{O}_{\phi}\left(x_{2}\right)\right\rangle \sim\left(\frac{1}{\sin ^{2} \ell_{2} / 2}\right)^{i m}+\text { subleading }
$$

where now $\ell_{2}$ is the geodesic length on $H_{2}$. At short distances this gives the short distance behavior $\ell_{2}^{2 i m}$ associated to a field of imaginary weight, as one usually obtains 38 in $\mathrm{dS} / \mathrm{CFT}$.

37 We should emphasize that the expression (E.5) has branch cuts in the complex $\ell_{2}$ plane, so in deriving (E.13) we have made a specific branch choice. This appearance of branch ambiguities is rather common when analytically continuing correlators in curved spacetime, and can be thought of as arising from the ambiguity of vacuum choice in cosmological spacetimes. In this case, there is a clear choice of branch prescription;we simply choose the branch which matches the usual short distance behavior of the standard AdS vacuum.

38 We should note that in terms of the boundary field theories, the analytic continuation from the CFT on $d S_{2}$ in ( $(\mathrm{E} .13)$ to the ECFT on $H_{2}$ given by ( $\left.\mathrm{E.14}\right)$ is $t \rightarrow t+i \pi / 2$. 


\section{References}

[1] E. W. Kolb and M. S. Turner, The Early Universe, Addison-Wesley, Redwood City (1990).

[2] A.D. Linde, Particle Physics and Inflationary Cosmology, Harwood, Chur, Switzerland (1990).

[3] A.R. Liddle and D.H. Lyth, Cosmological Inflation and Large-Scale Structure, Cambridge University Press, Cambridge, England (2000).

[4] R. Bousso and J. Polchinski, Quantization of four-form fluxes and dynamical neutralization of the cosmological constant, JHEP 0006, 006 (2000) [arXiv:hep-th/0004134].

[5] S. Kachru, R. Kallosh, A. Linde and S. P. Trivedi, De Sitter vacua in string theory, Phys. Rev. D 68, 046005 (2003) [arXiv:hep-th/0301240].

[6] F. Denef, M. Douglas, B. Florea, A. Grassi and S. Kachru, Fixing all moduli in a simple F-theory compactification, [arXiv:hep-th/0503124]; F. Denef, M. Douglas and B. Florea, Building a better racetrack, JHEP 0406 (2004) 034 [arXiv:hep-th/0404257; P. S. Aspinwall and R. Kallosh, Fixing all moduli for M-theory on K3 $x$ K3, [arXiv:hepth/0506014.

[7] J. Conlon, F. Quevedo and K. Suruliz, Large-volume flux compactifications: Moduli spectrum and D3/D7 soft supersymmetry breaking, [arXiv:hep-th/0505076]; P. Berglund and P. Mayr, Non-perturbative superpotentials in F-theory and string duality, [arXiv:hep-th/0504058]; V. Balasubramanian, P. Berglund, J. Conlon and F. Quevedo, Systematics of moduli stabilisation in Calabi-Yau flux compactifications, JHEP 0503 (2005) 007 [arXiv:hep-th/0502058].

[8] A. Saltman and E. Silverstein, A new handle on de Sitter compactifications, [arXiv:hepth/0411271]; A. Maloney, E. Silverstein and A. Strominger, de Sitter space in noncritical string theory, [arXiv:hep-th/0205316]. B. Acharya, A moduli fixing mechanism in M-theory, [arXiv:hep-th/0212294]. B. de Carlos, A. Lukas and S. Morris, Nonperturbative vacua for M-theory on G2 manifolds, JHEP 0412 (2004) 018 [arXiv:hepth/0409255.]

[9] G. Curio, A. Krause and D. Lüst, Moduli stabilization in the heterotic/IIB discretuum, [arXiv:hhep-th/0502168]; S. Gurrieri, A. Lukas and A. Micu, Heterotic on Half-flat, Phys.Rev. D70 (2004) 126009 [arXiv:hep-th/0408121]; K. Becker, M. Becker, K. Dasgupta, P. Green and E. Sharpe, Compactifications of Heterotic Strings on Non-Kahler complex manifolds II, Nucl.Phys. B678 (2004) 19 [arXiv:hep-th/0310058]; M. Becker, G. Curio and A. Krause, De Sitter Vacua from Heterotic M Theory, Nucl.Phys. B693 (2004) 223 [arXiv:hep-th/0403027]; R. Brustein and S.P. de Alwis, Moduli Potentials in String Compactifications with Fluxes: Mapping the Discretuum, Phys.Rev. D69 (2004) 126006 [arXiv:hep-th/0402088]; S. Gukov, S. Kachru, X. Liu and L. McAllister, Heterotic Moduli Stabilization with Fractional Chern-Simons Invariants, Phys.Rev. D69 
(2004) 086008 [arXiv:hep-th/0310159]; E. Buchbinder and B. Ovrut, Vacuum Stability in Heterotic M-theory, Phys.Rev. D69 (2004) 086010 [arXiv:hep-th/0310112]; G. Cardoso, G. Curio, G. Dall'Agata and D. Lüst, Heterotic string theory on nonKahler manifolds with H-flux and gaugino condensate, Fortsch. Phys. 52 (2004) 483 [arXiv:hep-th/0310021]; G. Cardoso, G. Curio, G. Dall'Agata and D. Lüst, BPS action and superpotential for heterotic string compactifications with fluxes, JHEP 0310 (2003) 004 [arXiv:hep-th/0306088].

[10] I. Antoniadis and T. Maillard, Moduli stabilization from magnetic fluxes in type I string theory, Nucl. Phys. B 716, 3 (2005) [arXiv:hep-th/0412008]; I. Antoniadis, A. Kumar and T. Maillard, Moduli stabilization with open and closed string fluxes, [arXiv:hep-th/0505260].

[11] O. DeWolfe, A. Giryavets, S. Kachru and W. Taylor, Type IIA moduli stabilization, JHEP 0507, 066 (2005) [arXiv:hep-th/0505160].

[12] L. Susskind, The anthropic landscape of string theory, arXiv:hep-th/0302219.

[13] T. Banks, Cosmological breaking of supersymmetry or little Lambda goes back to the future. II, arXiv:hep-th/0007146.

[14] T. Banks and W. Fischler, M-theory observables for cosmological space-times, arXiv:hepth/0102077.

[15] E. Witten, Quantum gravity in de Sitter space, arXiv:hep-th/0106109.

[16] W. Fischler, A. Kashani-Poor, R. McNees and S. Paban, The acceleration of the universe, a challenge for string theory, JHEP 0107, 003 (2001) [arXiv:hep-th/0104181].

[17] S. Hellerman, N. Kaloper and L. Susskind, String theory and quintessence, JHEP 0106, 003 (2001) [arXiv:hep-th/0104180].

[18] R. Bousso, Positive vacuum energy and the N-bound, JHEP 0011, 038 (2000) [arXiv:hep-th/0010252].

[19] A. Strominger, The dS/CFT correspondence, JHEP 0110, 034 (2001) [arXiv:hepth/0106113].

[20] G. L. Alberghi, D. A. Lowe and M. Trodden, Charged false vacuum bubbles and the AdS/CFT correspondence, JHEP 9907, 020 (1999) [arXiv:hep-th/9906047].

[21] J. M. Maldacena, The large $N$ limit of superconformal field theories and supergravity, Adv. Theor. Math. Phys. 2, 231 (1998) [Int. J. Theor. Phys. 38, 1113 (1999)] [arXiv:hep-th/9711200].

[22] E. Witten, Anti-de Sitter space and holography, Adv. Theor. Math. Phys. 2, 253 (1998) [arXiv:hep-th/9802150].

[23] S. S. Gubser, I. R. Klebanov and A. M. Polyakov, Gauge theory correlators from non-critical string theory, Phys. Lett. B 428, 105 (1998) [arXiv:hep-th/9802109].

[24] O. Aharony, S. S. Gubser, J. M. Maldacena, H. Ooguri and Y. Oz, Large N field theories, string theory and gravity, Phys. Rept. 323, 183 (2000) [arXiv:hep-th/9905111]. 
[25] S. K. Blau, E. I. Guendelman and A. H. Guth, The Dynamics Of False Vacuum Bubbles, Phys. Rev. D 35, 1747 (1987).

[26] E. Farhi and A. H. Guth, An Obstacle To Creating A Universe In The Laboratory, Phys. Lett. B 183, 149 (1987).

[27] E. Farhi, A. H. Guth and J. Guven, Is It Possible To Create A Universe In The Laboratory By Quantum Tunneling?, Nucl. Phys. B 339, 417 (1990).

[28] J. Maldacena and L. Maoz, Wormholes in AdS, JHEP 0402, 053 (2004) [arXiv:hepth/0401024].

[29] J. Louko, D. Marolf and S. F. Ross, On geodesic propagators and black hole holography, Phys. Rev. D 62, 044041 (2000) [arXiv:hep-th/0002111].

[30] P. Kraus, H. Ooguri and S. Shenker, Inside the horizon with AdS/CFT, Phys. Rev. D 67, 124022 (2003) [arXiv:hep-th/0212277].

[31] L. Fidkowski, V. Hubeny, M. Kleban and S. Shenker, The black hole singularity in AdS/CFT, JHEP 0402, 014 (2004) [arXiv: hep-th/0306170].

[32] J. M. Maldacena, Eternal black holes in Anti-de-Sitter, JHEP 0304, 021 (2003) [arXiv:hep-th/0106112].

[33] V. Balasubramanian, P. Kraus, A. E. Lawrence and S. P. Trivedi, Holographic probes of anti-de Sitter space-times, Phys. Rev. D 59, 104021 (1999) [arXiv:hep-th/9808017].

[34] T. Banks, Landskepticism or why effective potentials don't count string models, arXiv:hep-th/0412129.

[35] R. Bousso, Cosmology and the S-matrix, Phys. Rev. D 71, 064024 (2005) [arXiv:hepth/0412197].

[36] W. Israel, Thermo Field Dynamics Of Black Holes, Phys. Lett. A 57, 107 (1976).

[37] T. Banks, Heretics of the false vacuum: Gravitational effects on and of vacuum decay. II, arXiv:hep-th/0211160.

[38] L. Susskind, private communication.

[39] W. Israel, Singular Hypersurfaces And Thin Shells In General Relativity, Nuovo Cim. B 44S10, 1 (1966) [Erratum-ibid. B 48, 463 (1967 NUCIA,B44,1.1966)].

[40] A. Aguirre and M. C. Johnson, Dynamics and instability of false vacuum bubbles, arXiv: gr-qc/0508093.

[41] M. C. Johnson, private communication.

[42] J. Garriga and A. Vilenkin, Perturbations on domain walls and strings: A Covariant theory, Phys. Rev. D 44, 1007 (1991).

[43] J. Garriga and A. Vilenkin, Quantum fluctuations on domain walls, strings and vacuum bubbles, Phys. Rev. D 45, 3469 (1992).

[44] R. Emparan, AdS/CFT duals of topological black holes and the entropy of zero-energy states, JHEP 9906, 036 (1999) [arXiv:hep-th/9906040].

[45] V. Balasubramanian, V. Jejjala and J. Simon, The library of Babel, arXiv:hepth/0505123. 
[46] V. Balasubramanian, J. de Boer, V. Jejjala and J. Simon, The library of Babel: On the origin of gravitational thermodynamics, arXiv:hep-th/0508023.

[47] G. W. Gibbons and S. W. Hawking, Cosmological Event Horizons, Thermodynamics, And Particle Creation, Phys. Rev. D 15, 2738 (1977).

[48] T. Jacobson, On the nature of black hole entropy, arXiv:gr-qc/9908031.

[49] J. M. Maldacena and A. Strominger, AdS(3) black holes and a stringy exclusion principle, JHEP 9812, 005 (1998) [arXiv:hhep-th/9804085].

[50] L. Randall and R. Sundrum, A large mass hierarchy from a small extra dimension, Phys. Rev. Lett. 83, 3370 (1999) [arXiv:hep-ph/9905221].

[51] S. S. Gubser, AdS/CFT and gravity, Phys. Rev. D 63, 084017 (2001) [arXiv:hepth/9912001.

[52] T. Banks, M. R. Douglas, G. T. Horowitz and E. J. Martinec, AdS dynamics from conformal field theory, arXiv:hep-th/9808016.

[53] A. Hamilton, D. Kabat, G. Lifschytz and D. A. Lowe, Local bulk operators in AdS/CFT: A boundary view of horizons and locality, arXiv:hep-th/0506118.

[54] S. B. Giddings, The boundary S-matrix and the AdS to CFT dictionary, Phys. Rev. Lett. 83, 2707 (1999) [arXiv:hep-th/9903048].

[55] N. Birrell and P. Davies, Quantum Fields in Curved Space, Cambridge University Press, Cambridge (1982).

[56] E. Witten and S. T. Yau, Connectedness of the boundary in the AdS/CFT correspondence, Adv. Theor. Math. Phys. 3, 1635 (1999) [arXiv:hep-th/9910245].

[57] G. J. Galloway, K. Schleich, D. M. Witt and E. Woolgar, Topological censorship and higher genus black holes, Phys. Rev. D 60, 104039 (1999) [arXiv:gr-qc/9902061].

[58] G. J. Galloway, K. Schleich, D. Witt and E. Woolgar, The AdS/CFT correspondence conjecture and topological censorship, Phys. Lett. B 505, 255 (2001) [arXiv:hepth/9912119].

[59] R. Bousso, Holography in general space-times, JHEP 9906, 028 (1999) [arXiv:hepth/9906022].

[60] D. Bak, M. Gutperle and S. Hirano, A dilatonic deformation of AdS(5) and its field theory dual, JHEP 0305, 072 (2003) [arXiv:hep-th/0304129].

[61] A. B. Clark, D. Z. Freedman, A. Karch and M. Schnabl, The dual of Janus $((<:) \leftrightarrow$ $(:>)$ ) an interface CFT, Phys. Rev. D 71, 066003 (2005) [arXiv:hep-th/0407073].

[62] S. R. Coleman and F. De Luccia, Gravitational Effects On And Of Vacuum Decay, Phys. Rev. D 21, 3305 (1980).

[63] A. H. Guth and E. J. Weinberg, Could The Universe Have Recovered From A Slow First Order Phase Transition?, Nucl. Phys. B 212, 321 (1983).

[64] W. Fischler, D. Morgan and J. Polchinski, Quantum Nucleation Of False Vacuum Bubbles, Phys. Rev. D 41, 2638 (1990). 
[65] W. Fischler, D. Morgan and J. Polchinski, Quantization Of False Vacuum Bubbles: A Hamiltonian Treatment Of Gravitational Tunneling, Phys. Rev. D 42, 4042 (1990).

[66] A. D. Linde, Hard art of the universe creation (stochastic approach to tunneling and baby universe formation), Nucl. Phys. B 372, 421 (1992) [arXiv:hep-th/9110037].

[67] S. Aminneborg, I. Bengtsson, D. Brill, S. Holst and P. Peldan, Black holes and wormholes in 2+1 dimensions, Class. Quant. Grav. 15, 627 (1998) [arXiv:gr-qc/9707036].

[68] K. Krasnov, Holography and Riemann surfaces, Adv. Theor. Math. Phys. 4, 929 (2000) [arXiv:hep-th/0005106].

[69] G. T. Horowitz and D. Marolf, A new approach to string cosmology, JHEP 9807, 014 (1998) [arXiv:hep-th/9805207].

[70] A. Maloney, $A d S_{3}$ Cosmology, to appear. 